\title{
INFLUENCIA DE LA DUCTILIDAD DE LAS ARMADURAS DE ACERO EN EL COMPORTAMIENTO Y LA SEGURIDAD DE LOSAS DE HORMIGÓN ARMADO
}

\author{
(THE INFLUENCE OF STEEL REINFORCEMENT DUCTILITY IN THE BEHAVIOUR AND \\ SECURITY OF REINFORCED CONCRETE SLABS)
}

Honorino Ortega, Dr. Ingeniero de Caminos

Grupo SiderúrgicoCELSA

Fecha de recepción:12-VII-99

ESPAÑA

4543

\section{RESUMEN \\ Este trabajo se basa en los resultados y conclusiones básicas de la tesis doctoral del autor, dirigida por el Profesor D. José Calavera.}

Se propone, por un lado, una forma de medir mediante un único parámetro la ductilidad de los aceros utilizados como armaduras para hormigón armado, de forma que se puedan comparar y clasificar con arreglo al mismo.

Por otro, se estudian los resultados de los ensayos sobre 12 losas continuas de dos vanos, de sección maciza, armadas con cuatro cuantias diferentes elegidas de forma que, en la sección más solicitada, sea el acero el que alcance, en primer lugar, su agotamiento (situación en el dominio 2, pivote A). El armado para conseguir las citadas cuantías se realiza con aceros de la misma resistencia nominal y del mismo diámetro, pero con distinta ductilidad. Los resultados de este estudio experimental, ponen de manifiesto la importancia de la ductilidad del acero en relación con el comportamiento, asi como con la seguridad real de la estructura frente al colapso y proporciona un conjunto de conclusiones y propuestas en relación con este interesante tema.

\section{SUMMARY}

This work is based on the results and basic conclusions of author's doctoral thesis, directed by Professor José

Calavera.

On the one hand, a new form to messure the ductility of those steels used like reinforcement of concrete, is proposed, in order to be compared and clasified in accordancer with this one.

On the other hand it has been studied the results of the tests on 12 continuous slabs of two equal spans, reinforced with four different steel types choosen so that, in the more requested section the steel will reach, in first place, its collapse (situation in the 2 domain, $A$ pivot). The reinforcement to allow the mentioned extent, is provided with same nominal resistance steels and same diameter, but with different ductility. The results of this experimental study, reveals the importance of the steel ductility concerning the behaviour and real security of the structure in front of the collapse, and furnish a group of conclusions and proposals concerning this interesting subject.

\section{Introducción}

Siempre que se trata sobre la capacidad de redistribución o la seguridad frente al colapso de una estructura se implica la consideración de su comportamiento no lineal.
Está generalmente admitido que fueron dos los caminos que, hace más de un siglo, condujeron al inicio del estudio del comportamiento no lineal, a veces llamado plástico, de los materiales y, por ende, de las estructuras. Por un lado, se observó cómo, en la práctica, la capacidad 
resistente de piezas hiperestáticas de acero aumentaba, de forma muy considerable, en relación con la obtenida a través de los principios de la teoría lineal, a veces también llamada elástica.

Por otro lado, se comprobó, en estas mismas estructuras hiperestáticas, cómo, a partir de un momento dado, las deformaciones que se obtenían eran muy importantes para pequeños incrementos de la carga, apartándose del comportamiento lineal previsto.

De forma casi espontánea se pensó en que un cierto fenómeno de fluencia o plastificación debía producirse en el material para justificar este apartamiento del comportamiento previsto.

Así, a través de los años se fueron conociendo cada vez con más precisión estos fenómenos y su formulación matemática se hizo realidad ( Tresca, St. Venant, Levy, Von Mises, Prandtl, Melan, Prager, Kacinzy, Bleich, Baker, Thurlimann y tantos otros que estudiaron el comportamiento no lineal en el campo de las estructuras metálicas). En la técnica del hormigón armado, los estudios sobre el comportamiento no lineal se retrasan considerablemente hasta después de acabada la Segunda Guerra Mundial y sólo a partir de 1953 se inicia un fuerte impulso de su investigación en el seno del CEB, auspiciada, entre otros, por los Profesores A.L.L. Baker, Macchi y Guyon.

Al ser el hormigón armado un material compuesto, se hizo preciso estudiar en profundidad, por un lado, el comportamiento bajo distintas situaciones de cada material por separado (hormigón y acero)y, por otro, las interacciones entre ambos, una vez unidos, para llegar a conocer el comportamiento del compuesto hormigón armado.

En las primeras investigaciones sobre el comportamiento no lineal de elementos de hormigón armado, se supuso que el hormigón era el causante en todos los casos del colapso, ya que al acero se le suponía ductilidad suficiente para no limitar las rotaciones de las rótulas plásticas formadas. Así, en el Código Modelo del 78, se adopta para la relación rotaciones $-\mathrm{x} / \mathrm{d}$, un esquema en el que el acero no interviene en la capacidad de rotación.

Investigaciones posteriores, sobre todo en Alemania (Eligehausen, Langer, Eibl, Curbach y Stempniewski, entre otros) manifiestan el diferente comportamiento en régimen no lineal en función de las características de ductilidad del acero empleado como armadura. Con base en estas investigaciones se modifica la curva rotaciones - $\mathrm{x} / \mathrm{d}$ en el Código Modelo de 1990 y se adopta una en la que el tipo de acero, en función de su ductilidad, influye en la capacidad de rotación plástica máxima admisible.

En España y con la colaboración de la Asociación de Trefiladores del Acero, se realiza un estudio experimen- tal, llevado a cabo por el Laboratorio Central de Intemac sobre el comportamiento de losas hiperestáticas (de dos vanos iguales) armadas con aceros de distintos niveles de ductilidad (muy bajo, bajo y alto) con el fin de estudiar la relación entre la ductilidad del acero y la capacidad de redistribución real alcanzada en las losas.

Estos ensayos fueron utilizados, como se ha dicho con anterioridad, en la tesis doctoral de Honorino Ortega, dirigida por el Prof. D. José Calavera.

\section{Objetivos de este trabajo}

Tres son sus objetivos básicos:

a) Concepto de ductilidad aplicado a las armaduras. Propuesta de valoración de la ductilidad a través de los conceptos del factor de energía plástica y del factor de ductilidad.

b) Estudio de la capacidad de redistribución de los elementos considerados, en función del nivel de ductilidad del acero utilizado en su armado.

c) Valoración de la seguridad real frente al colapso, en función de la ductilidad del acero para los elementos estructurales considerados.

\section{Objetivo a)}

De una forma sencilla, podemos definir la ductilidad como la capacidad de un acero para admitir deformaciones importantes bajo tensiones superiores a su límite elástico. Aunque es un concepto que se entiende con facilidad, su valoración numérica no es tan sencilla.

La forma de definir la ductilidad de una armadura se ha basado en la consideración de dos parámetros obtenibles a partir de la curva tensiones-deformaciones del acero: el alargamiento bajo carga máxima, $\varepsilon_{\text {máx }}$ y la relación $\mathbf{f}_{s} / \mathbf{f}_{\mathbf{y}}$, llamada también endurecimiento, entre la tensión máxima $\left(\mathbf{f}_{\mathrm{s}}\right)$ y la tensión correspondiente al límite elástico (f) . La ductilidad es mayor cuanto mayor son los parámetros citados pero, al ser parejas de valores, no es posible mediante ellos cuantificar de forma unívoca el nivel de ductilidad. Por otro lado, pueden darse situaciones en las que los valores de los parámetros no permiten comparar ni graduar distintos aceros en relación con esta característica.

En este trabajo se proponen dos métodos para cuantificar la ductilidad y clasificar los aceros de acuerdo con un único parámetro, como se expone en el apartado 3.

\section{Objetivo b)}

En el programa experimental se ensayaron, como ya se ha 
apuntado, 12 losas armadas con distintas cuantías de armado y aceros de diferentes ductilidades, de acuerdo con el modelo descrito en el apartado 4. Se estudian, desde distintos puntos de vista, los resultados obtenidos en los ensayos hasta el colapso, llevados a cabo sobre dichas estructuras, al relacionarse las capacidades de redistribución máximas realmente obtenidas, con el nivel de ductilidad de las armaduras empleadas. En el apdo. 6 se desarrolla este punto. Como es lógico, las cuantías adoptadas en los armados se limitan dentro del dominio en el cual el acero es el material que alcanza, en primer lugar, el agotamiento por flexión simple.

\section{Objetivo c)}

Se estudia también otro tema de mucho interés, como es el referente a la relación entre la ductilidad del acero y la seguridad real frente al colapso del elemento estructural. Para ello, se comparan las cargas reales de ensayo con las teóricas obtenidas mediante la aplicación de las fórmulas habitualmente empleadas en la comprobación de secciones de hormigón estructural. En este sentido, se han aplicado las fórmulas aproximadas de comprobación contenidas en la Instrucción EH -91 y en la nueva EHE de 1999. En el apartado 7 se exponen estos aspectos.

\section{La ductilidad de las armaduras para hormigón}

Una de las formas que el autor ha propuesto para valorar la ductilidad de las armaduras es mediante la cuantificación de la energía plástica disponible en el acero, constituida por el área encerrada por la curva tensiones-deformaciones y el eje de deformaciones, tomando como abscisa límite superior la correspondiente al alargamiento bajo carga máxima $\varepsilon_{\text {máx }}$, según queda expuesto en la figura 1 .

Esta forma de valorar la ductilidad proporciona una solución para su clasificación, como queda reflejado en la figura 2 mediante el denominado Factor de Energía Plástica (F.E.P.) que viene a representar el valor de la citada energía disponible en el acero.

Otra forma de considerar el valor de la ductilidad, es mediante el área encerrada bajo la curva tensiones - deformaciones, la recta paralela al eje x (de deformaciones) por el límite elástico y la recta paralela al eje y (de tensiones) por el valor de $\varepsilon_{\text {máx }}$ (ver figura 3 ). Es el parámetro que se ha denominado Factor de Ductilidad (F.D.).

Aplicando esta otra forma de afrontar el problema, llegamos a una clasificación de los aceros por su nivel de ductilidad que se representa en la figura 4.

\section{FACTOR DE ENERGÍA PLÁSTICA}

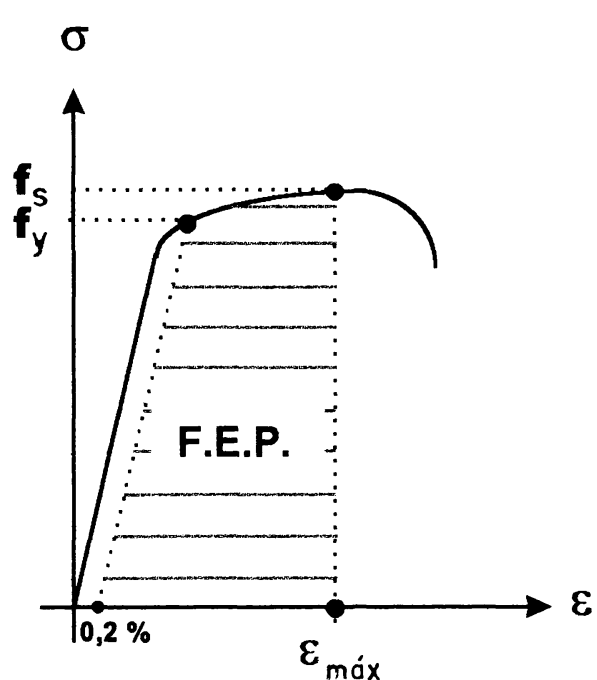

ACERO TREFILADO

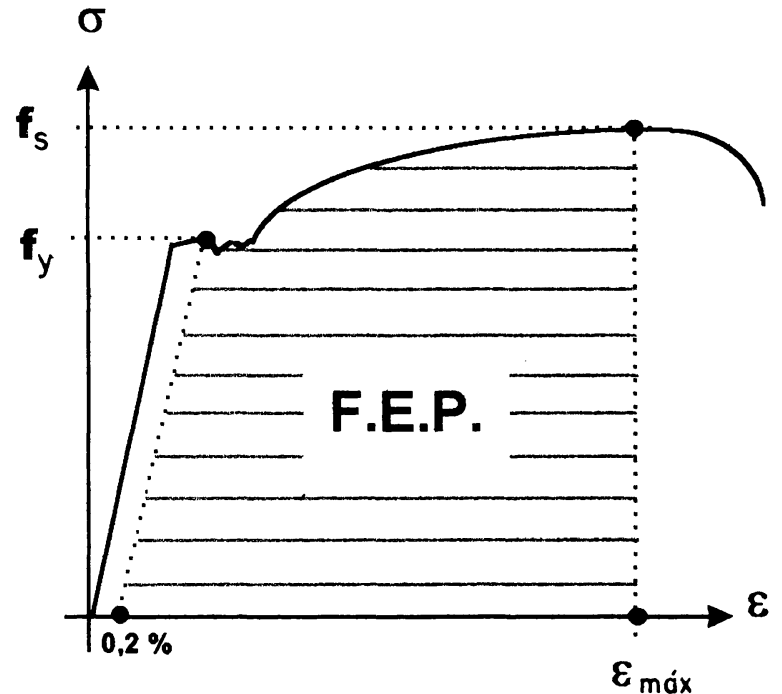

ACERO LAMINADO EN CALIENTE 


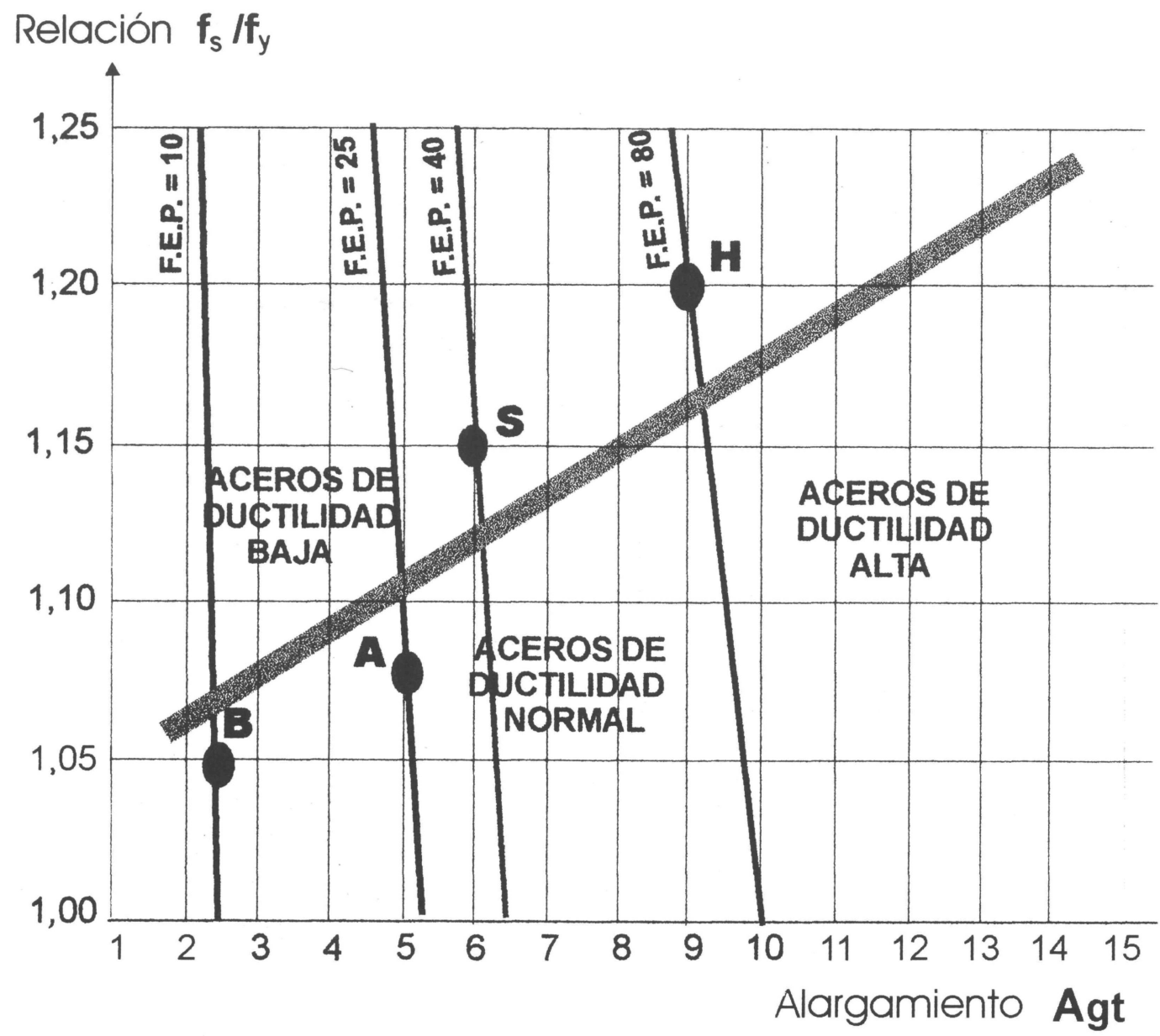

Figura 2.- Clasificación mediante el Factor de Energía Plástica (FEP) de aceros de $f_{y}=500 \mathrm{MPa}$.

\section{FACTOR DE DUCTILIDAD}

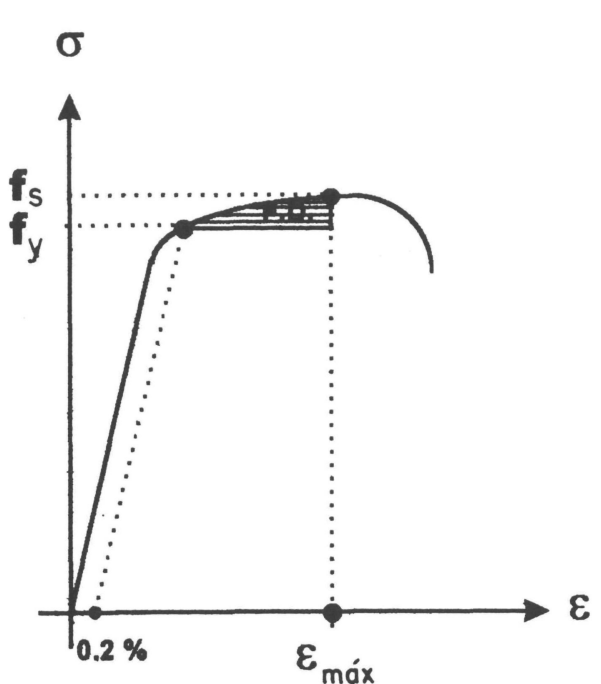

ACERO TREFILADO $\sigma$

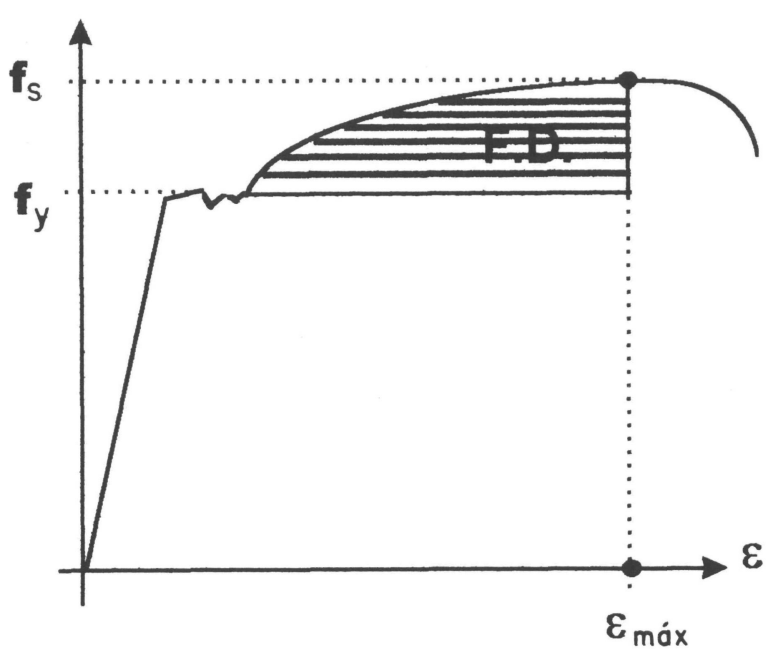

ACERO LAMINADO EN CALIENTE 


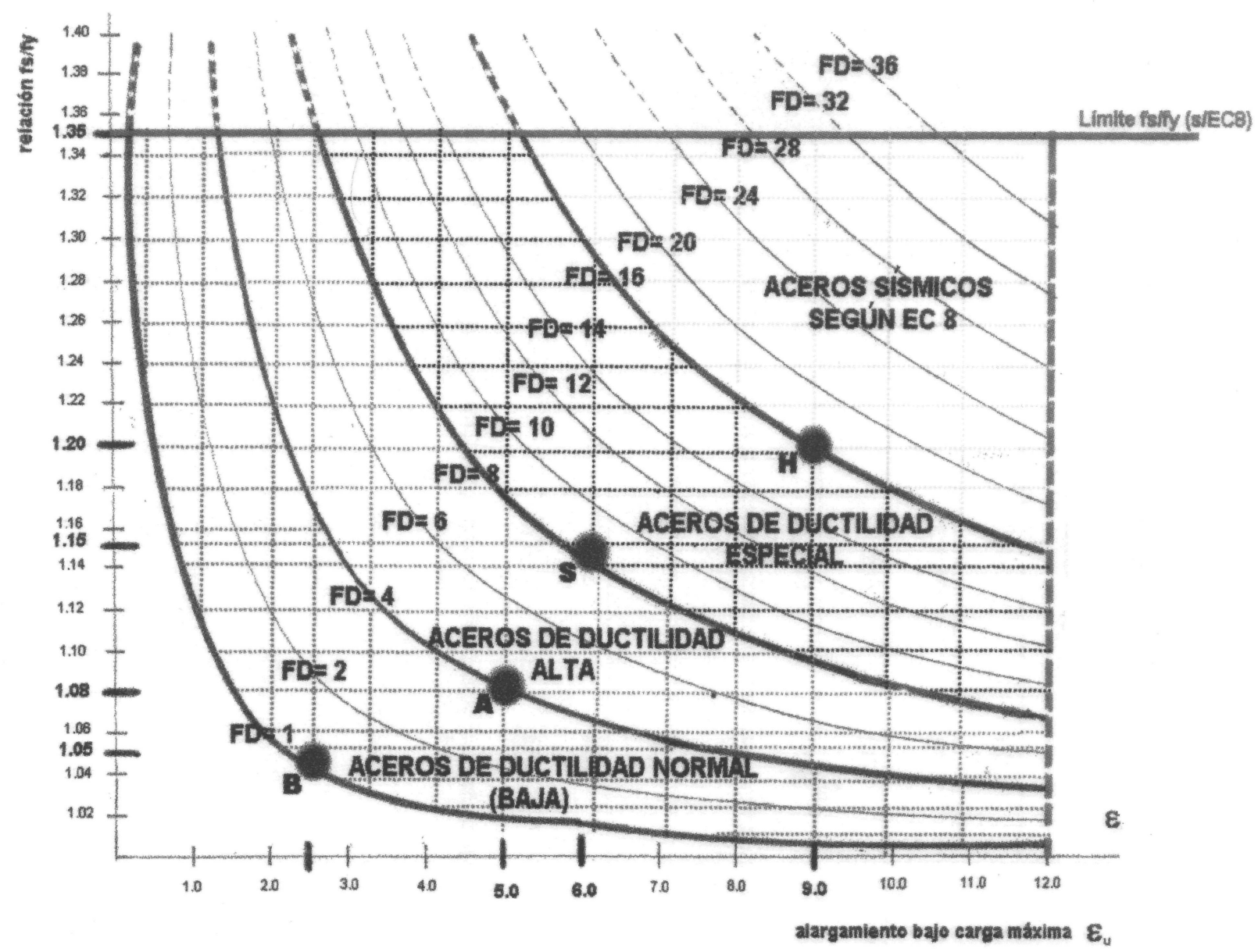

Figura 4.- Factor de ductilidad. Curvas de equiductilidad. Tipos de aceros.

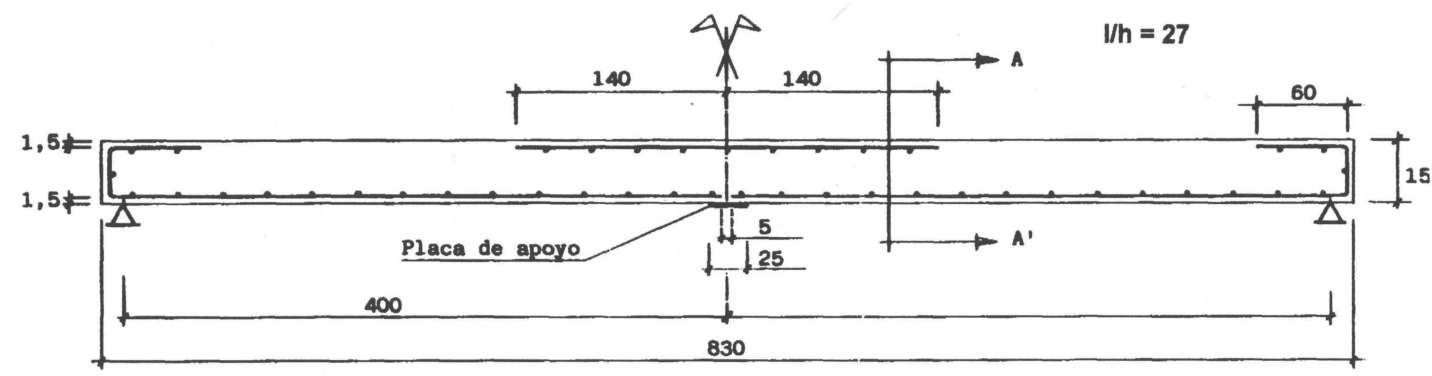

SECCIÓN LONGITUDINAL

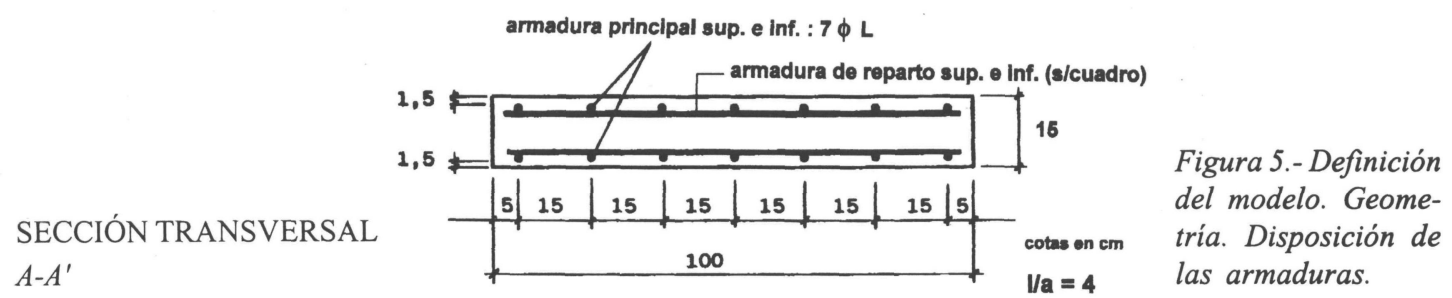

En ambos casos, ya sea mediante el factor de energía plástica o mediante el factor de ductilidad, se pueden valorar y comparar de una manera unívoca las ductilidades de las armaduras conociendo, a través de su curva tensiones-deformaciones, los valores de la relación $f_{s} / f_{y} y$ del alargamiento $\varepsilon_{\text {máx }}$, valores que, en todos los casos deben ser declarados y garantizados por el fabricante del acero en cuestión.

(c) Consejo Superior de Investigaciones Científicas Licencia Creative Commons 3.0 España (by-nc)

\section{Descripción del modelo estructural elegido en el estudio experimental}

El modelo estructural adoptado en los ensayos es el de una losa maciza, de sección uniforme, continua, de dos vanos iguales. En la figura 5 se representa el esquema de dicho modelo.

http://informesdelaconstruccion.revistas.csic.es 
CUADRO I.- Características de los materiales.

HORMIGÓN

\begin{tabular}{|c|}
\hline LOSA \\
\hline 1 \\
\hline 2 \\
\hline 3 \\
\hline 4 \\
\hline \hline 5 \\
\hline 6 \\
\hline 7 \\
\hline 10 \\
\hline 11 \\
\hline 12 \\
\hline
\end{tabular}

\begin{tabular}{|c|}
\hline $\begin{array}{c}\text { RESISTENCIAS } \\
\text { A COMPRESIÓN } \\
\text { MPa (1) }\end{array}$ \\
\hline 22,0 \\
\hline 20,3 \\
\hline 26,5 \\
\hline 30,8 \\
\hline \hline 26,1 \\
\hline 19,7 \\
\hline 26,6 \\
\hline 21,4 \\
\hline 17,6 \\
\hline 17,1 \\
\hline 17,5 \\
\hline 23,7 \\
\hline
\end{tabular}

AR M A D URAS

\begin{tabular}{|c|c|c|c|c|}
\hline $\begin{array}{l}\text { ALARG. } \\
\text { ROTURA } \\
\text { A5 (\%) (2) }\end{array}$ & $\begin{array}{c}\varepsilon_{u}(\text { Agt }) \\
(\%) \\
(2)\end{array}$ & $\begin{array}{r}\mathbf{f}_{\mathrm{s}} / \mathrm{f}_{\mathrm{y}} \\
(2)\end{array}$ & $\begin{array}{l}\text { LÍMITE } \\
\text { ELÁSTICO fy } \\
\operatorname{MPa}(2)\end{array}$ & $\begin{array}{c}\text { CARGA DE } \\
\text { ROTURA fs } \\
\text { MPa (2) }\end{array}$ \\
\hline 11,5 & 1,0 & 1,05 & 634,8 & 653,7 \\
\hline 12,0 & 1,5 & 1,06 & 638,3 & 676,2 \\
\hline 10,5 & 1,6 & 1,02 & 608,7 & 619,1 \\
\hline 8,8 & 0,9 & 1,00 & 597,5 & 599,5 \\
\hline$\overline{11,4}$ & $\overline{1,8}$ & $\overline{11,07}$ & 607,7 & 651,0 \\
\hline 11,7 & 1,8 & 1,04 & 610,9 & 635,6 \\
\hline 14,0 & 3,2 & 1,04 & 597,1 & 619,6 \\
\hline 11,2 & 3,2 & 1,06 & 542,6 & 575,0 \\
\hline$\overline{27,9}$ & $\overline{11,6}$ & $\overline{1,14}$ & 563,8 & $\overline{640,6}$ \\
\hline 22,9 & 10,1 & 1,12 & 590,3 & 660,9 \\
\hline 24,1 & 9,8 & 1,11 & 600,3 & 665,7 \\
\hline 23,7 & 10,5 & 1,13 & 599,2 & 679,2 \\
\hline
\end{tabular}

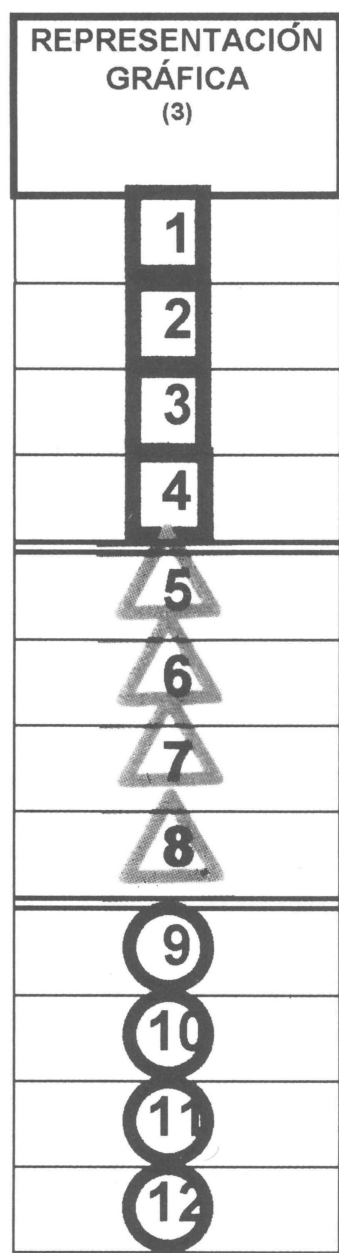

ACERO TREFILADO CON TRATAMIENTO ESPECIAL PARA OBTENER MUY BAJA DUCTILIDAD

ACERO TREFILADO NORMAL B500T EMPLEADO HABITUALMENTE EN MALLAS ELECTROSOLDADAS

2) Valores medios de los resultados de los ensayos realizados sobre la arm. principal (ver tablas de resultados)

(3) Estos símbolos se utilizarán en lo sucesivo para representar a cada losa en gráficos, cuadros, etc.

(c) Consejo Superior de Investigaciones Científicas Licencia Creative Commons 3.0 España (by-nc) 
En relación con las armaduras, se dispone la misma sección de acero en vanos y apoyo, lo que implica asumir la máxima redistribución admitida en la Instrucción de Forjados EF 96 y que, en porcentaje, supone un grado muy elevado de redistribución (del orden del 30\%).

Para conseguir los niveles de ductilidad previstos en las armaduras, fue preciso realizar un trabajo importante de clasificación de los aceros disponibles, así como el tratamiento de algunos de ellos para conseguir los niveles de ductilidad requeridos.

En el cuadro I se reflejan los valores reales de las características de los materiales empleados en cada losa. En el caso de los aceros, los resultados reflejados responden a la media de, al menos, diez ensayos efectuados sobre el material introducido como armadura en cada una de las piezas ensayadas.
La tipología de las cargas aplicadas hasta colapso se recoge en la figura 6. Con ella se consigue simular, con una desviación mínima (inferior al $2 \%$ ) las solicitaciones de una sobrecarga uniformemente repartida, que es la que, de forma habitual, se considera en los cálculos de estas estructuras.

En la figura 7 se refleja la instrumentación adoptada en los ensayos, que, como puede verse, es muy sencilla ya que el conocimiento de las solicitaciones en cada estado de carga se basa en la medición, fácil, fiable y poco costosa de la reacción hiperestática sobre el apoyo central,

\section{Resultados obtenidos}

En todos los casos, las losas se ensayaron hasta colapso. Como ya se ha dicho, en los ensayos se previó la medida de la reacción hiperestática en cada estado de carga para calcular con ella las solicitaciones reales hasta el colapso.

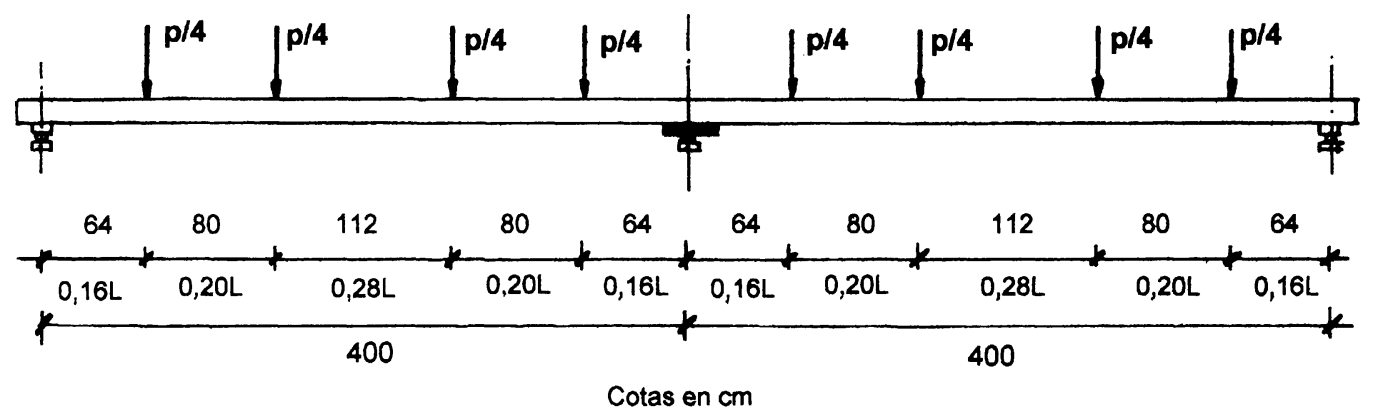

Figura 6.- Definición del modelo. Esquema de cargas.

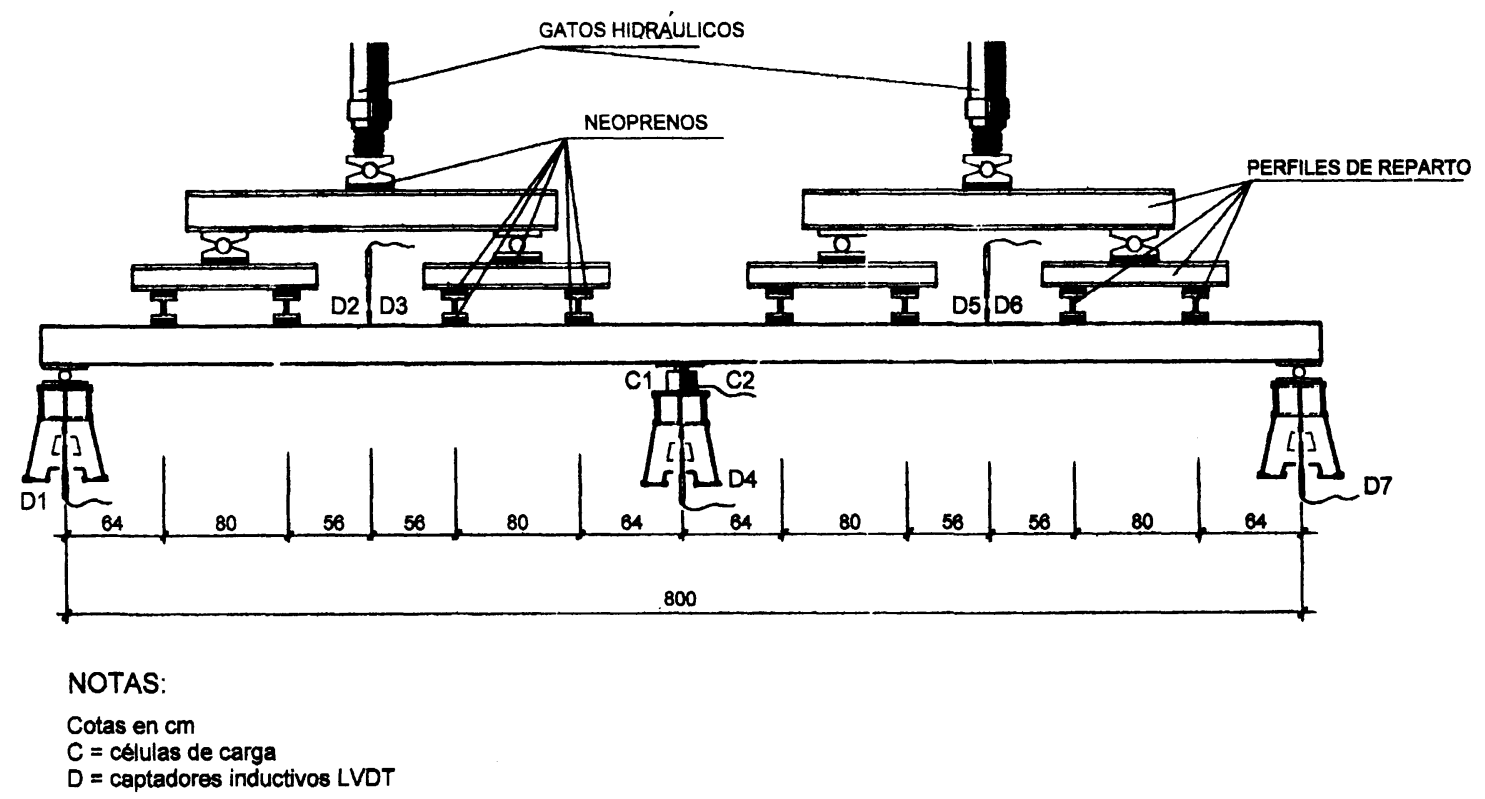

Figura 7.- Disposición del ensayo e instrumentación. 
En el cuadro II se exponen los valores de las cargas de colapso obtenidas en cada losa, así como los momentos máximos alcanzados para las mismas en apoyo y vano.

Además, se controlaron y midieron otras variables (flechas, fisuración, etc.) que manifiestan también unos resultados muy interesantes, pero fuera del entorno del tema que nos ocupa.

De forma fundamental interesan las relaciones entre cargas y esfuerzos y, sobre todo, las relaciones reales entre cargas y momentos flectores para las secciones de apoyo y de centro de vano.
Como se ha expuesto más arriba, para cada losa se obtiene la carga total máxima en ensayo alcanzada en el colapso, que se ha denominado carga real de colapso $\left(\mathrm{P}_{\text {real }}\right)$ y que se comparará con la carga teórica de agotamiento por flexión simple $\left(\mathrm{P}_{\text {teor }}\right)$ considerada como aquélla que produce, en la sección más solicitada de la estructura, el momento flector obtenido a través de las fórmulas de comprobación habitualmente empleadas en el cálculo de secciones, introduciendo en las mismas los valores reales de las distintas características de dicha sección más solicitada del modelo ensayado (cuantía real de acero introducida, valor real del límite elástico, valor real de la resistencia del hormigón, etc.).

CUADRO II

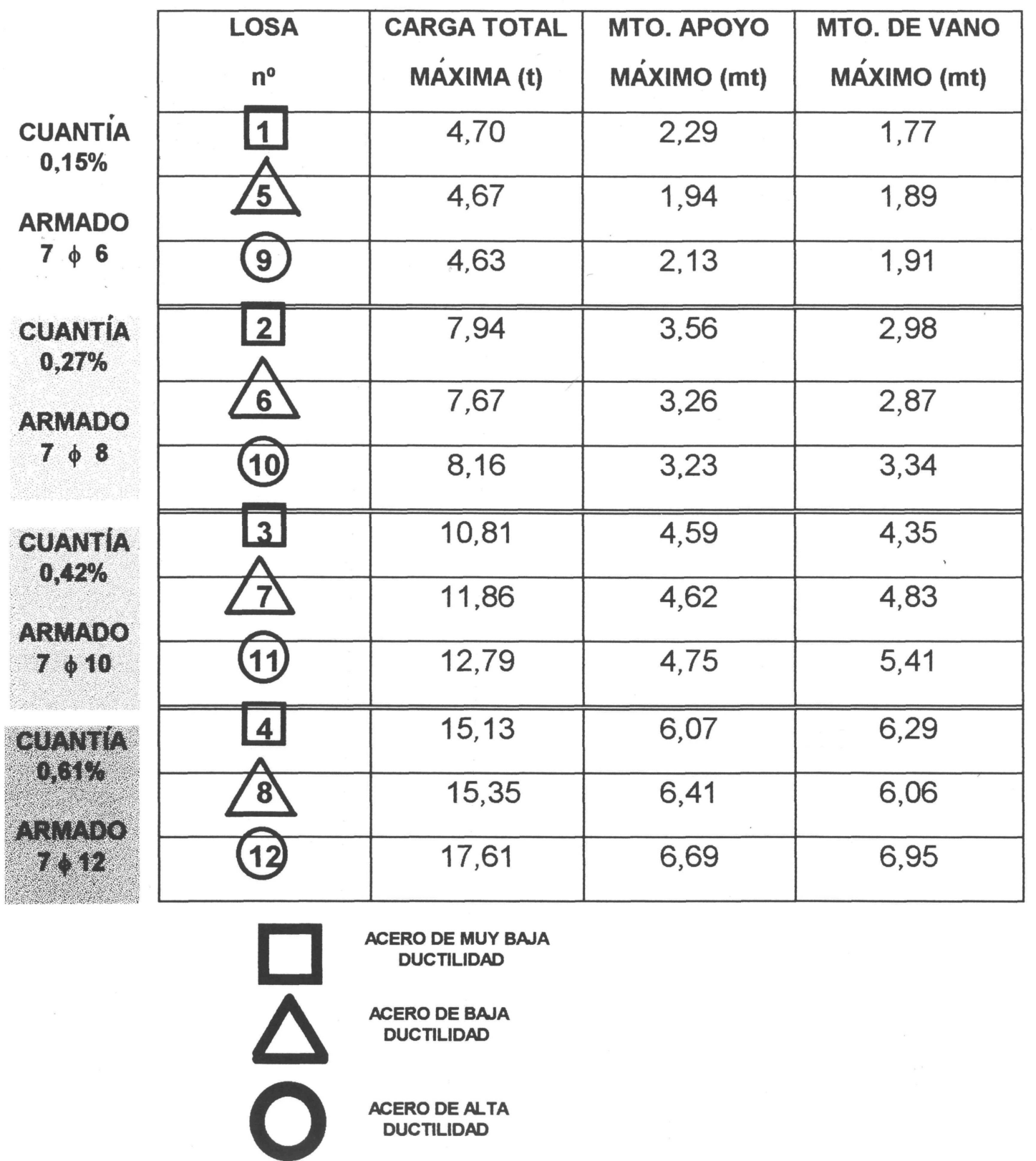




\section{Relación ductilidad vs. redistribución máxima}

En las figuras 8 a 11 se exponen los gráficos correspondientes a las relaciones cargas-momentos obtenidas experimentalmente para los doce casos ensayados, agrupados de tres en tres en función de la cuantía de acero.
Del análisis de estos diagramas y mediante la aplicación del concepto de redistribución en ensayo expuesto en la figura 12 , se obtienen las redistribuciones máximas realmente ocurridas en cada caso.

Si se comparan los valores de las redistribuciones obteni-

Los,

nEmetmanción En EasaYo

mantrems $y$ y

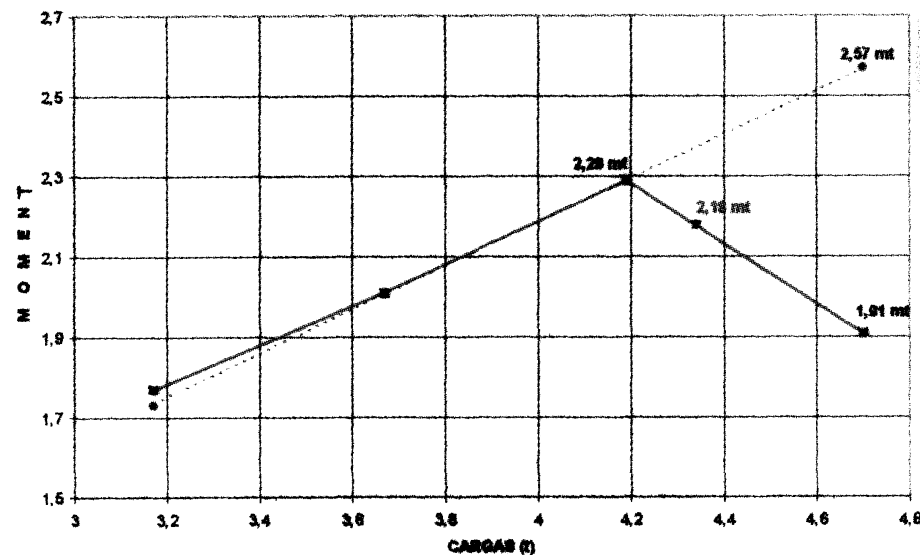

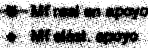

- asomk

of 1 = 0,89

Losa

ReDistruaucid' En Exay HuPó TES i

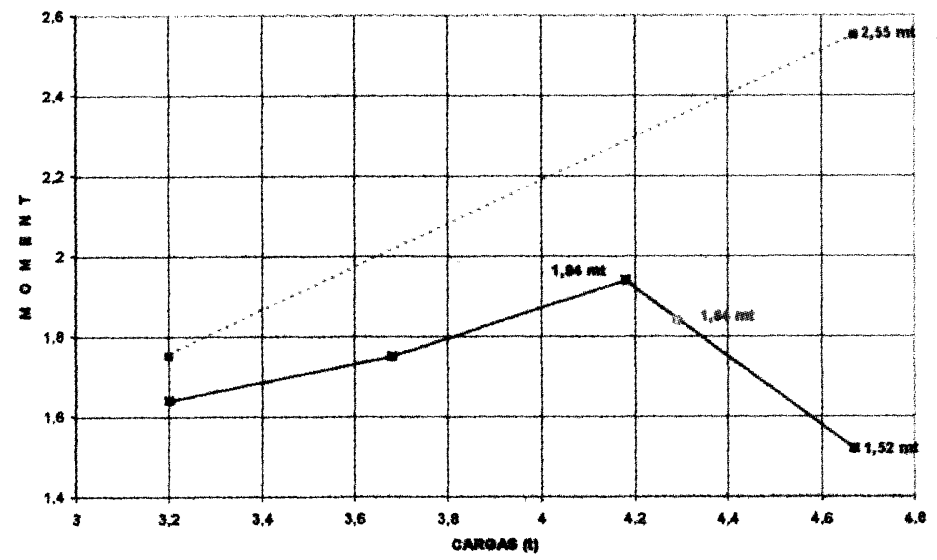

- Mar iner an apoyo - Mit olist. apoyo 0.86 mant

$81=0,76$

$\cos$

Repisthaución Eu Eusayo Hipótests iy
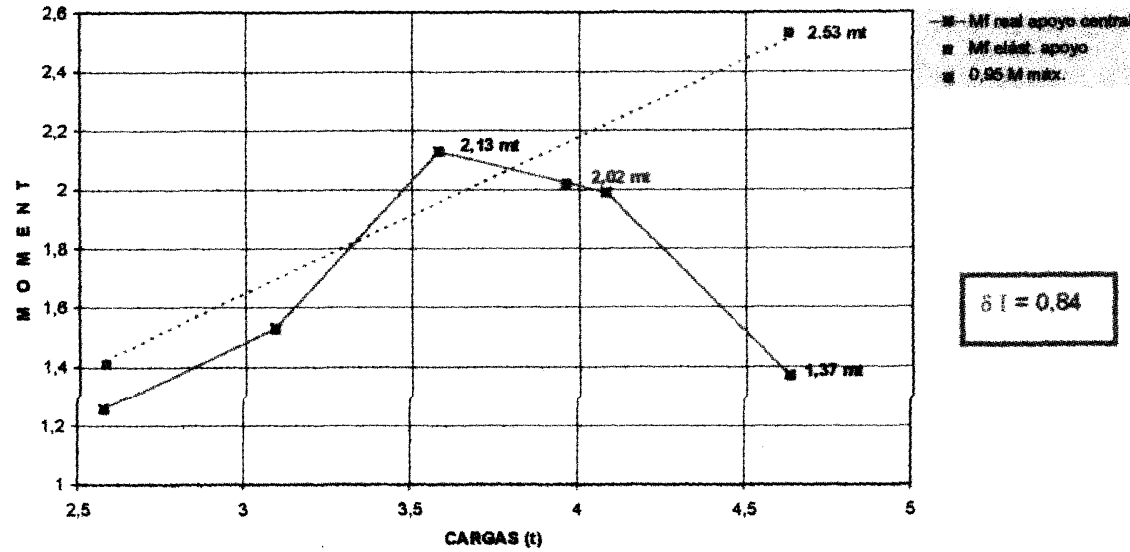

Figura 8.- Comparación redistribución obtenida en ensayo en losas de igual armado con aceros de distinta ductilidad. ARMADO $7 \phi 6 \mathrm{~mm}$. 
$\operatorname{Los} 2$

REDistravecion EN ENSAYO

Hiót TEss IY

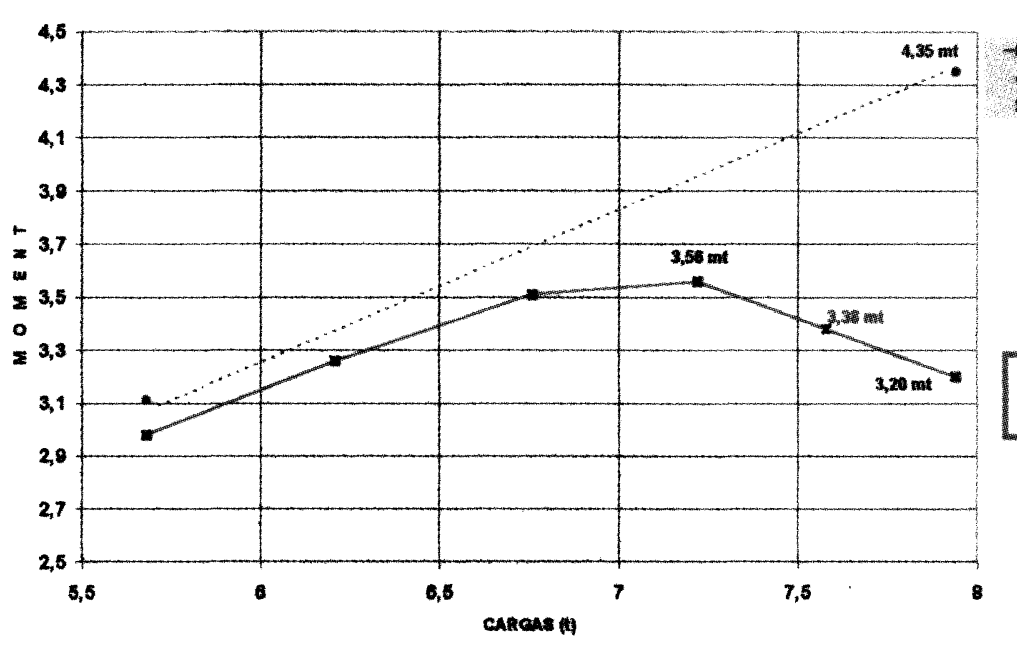

81.22

LOaA 6

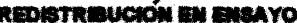

minóteses iy

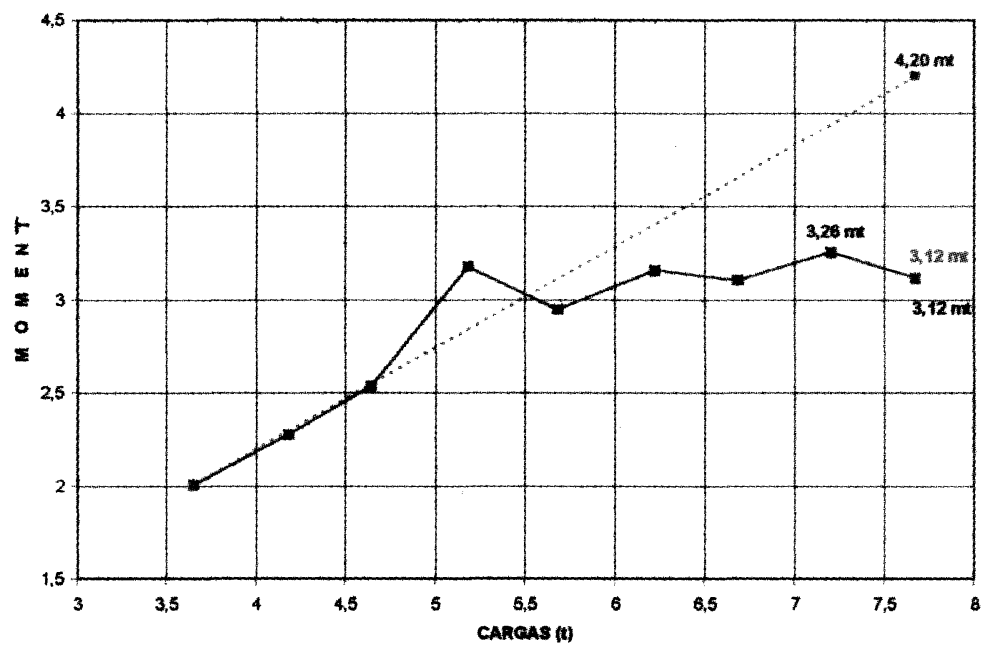

- Mit real apoyo central
- Mit elast apoyo

- 0,95 Mndix.

$8 I=0,78$

Taución EN ENSAYO

HPÓTES: I y

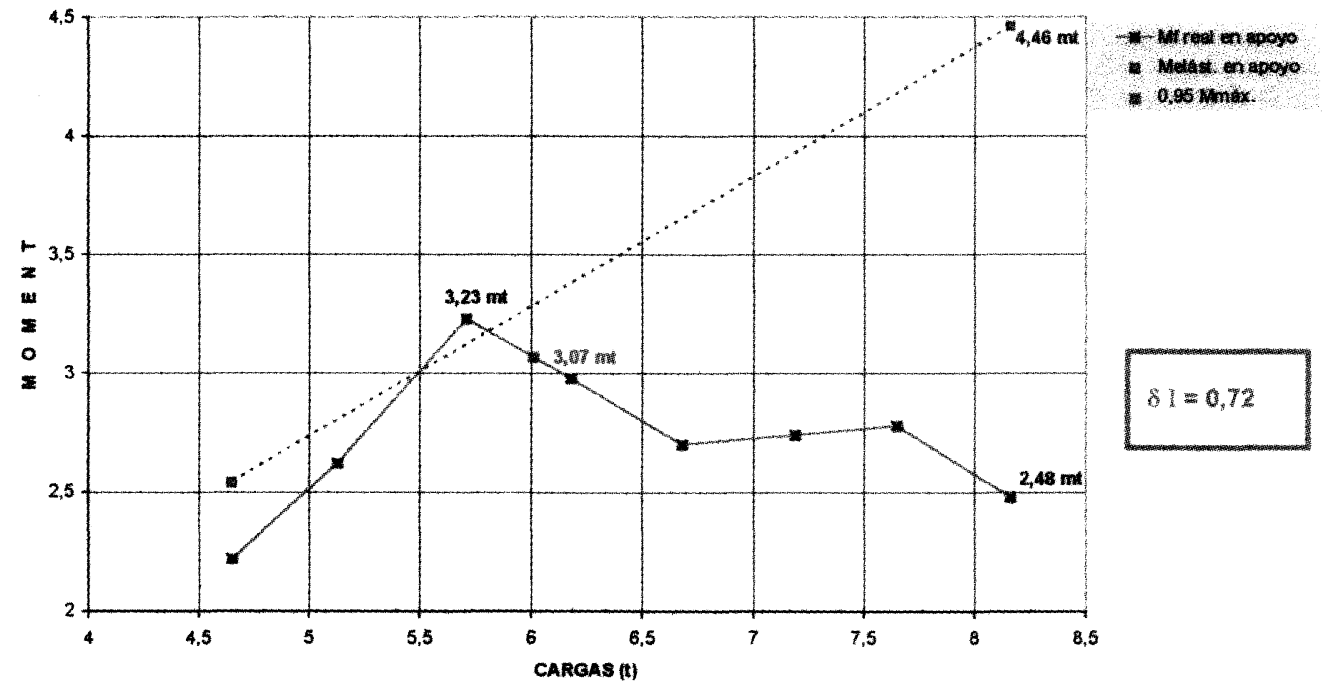

Figura 9.- Comparación redistribución obtenida en ensayo en losas de igual armado con aceros de distinta ductilidad. ARMADO $7 \phi 8 \mathrm{~mm}$. 


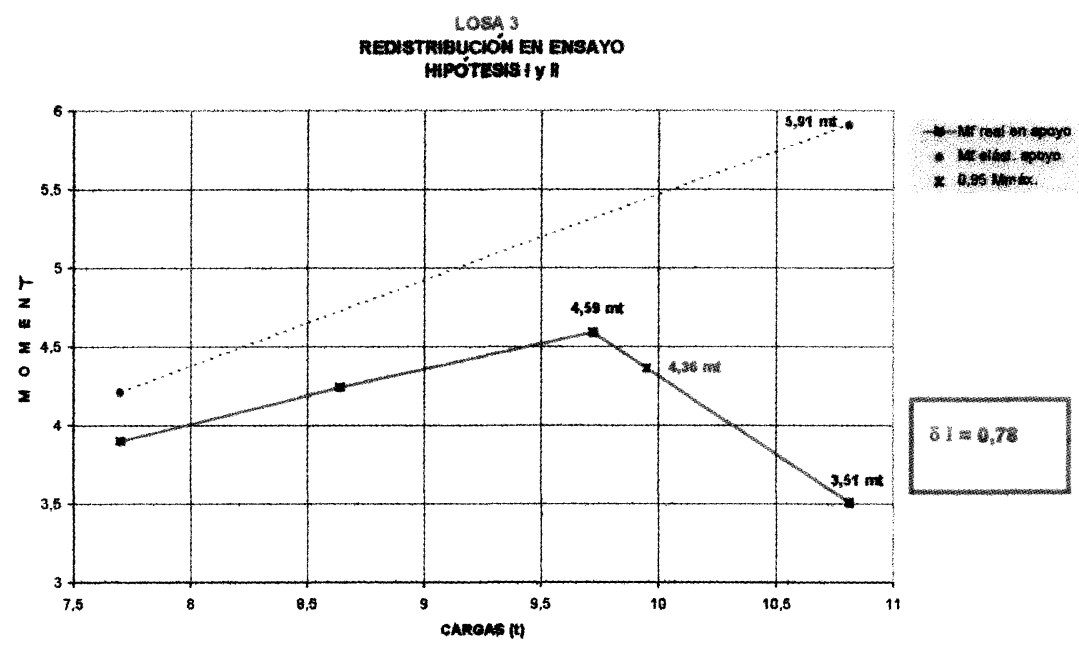

$\operatorname{LOSA} 7$

REDISTRBDUCIÓN EN ENSAYO

HIPÓTESIS IY II

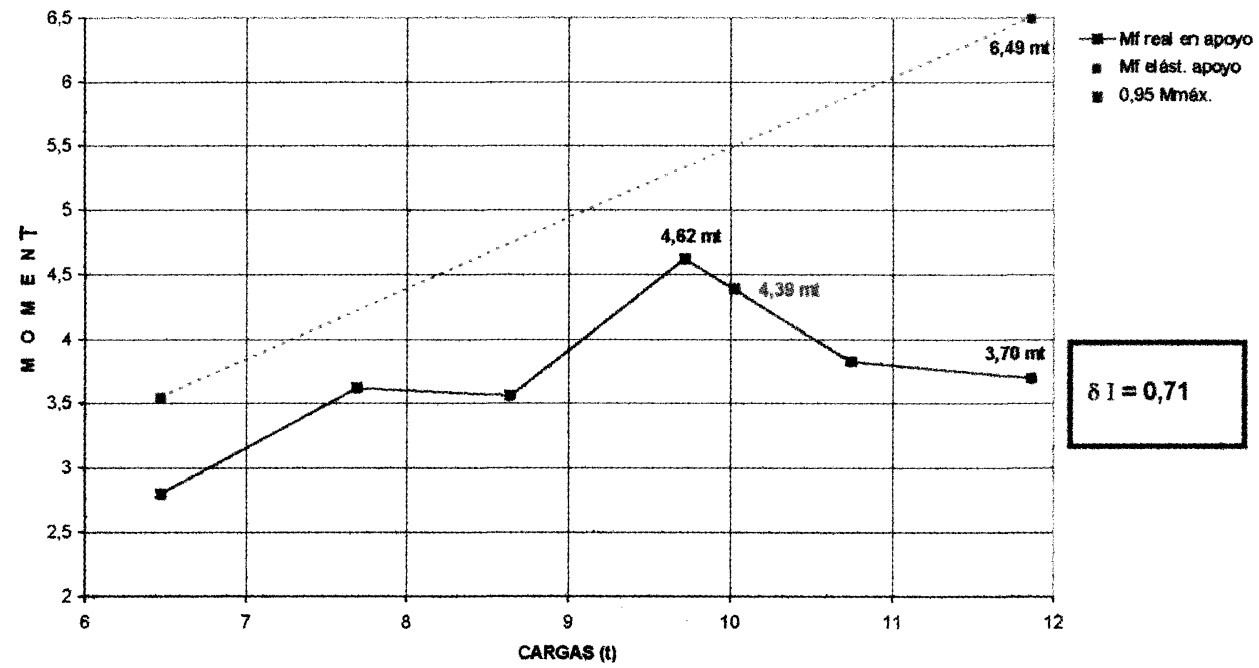

LOSA

REDISTRIBUCIÓN EN ENSAYO

HIPOTTESIS I y \|I

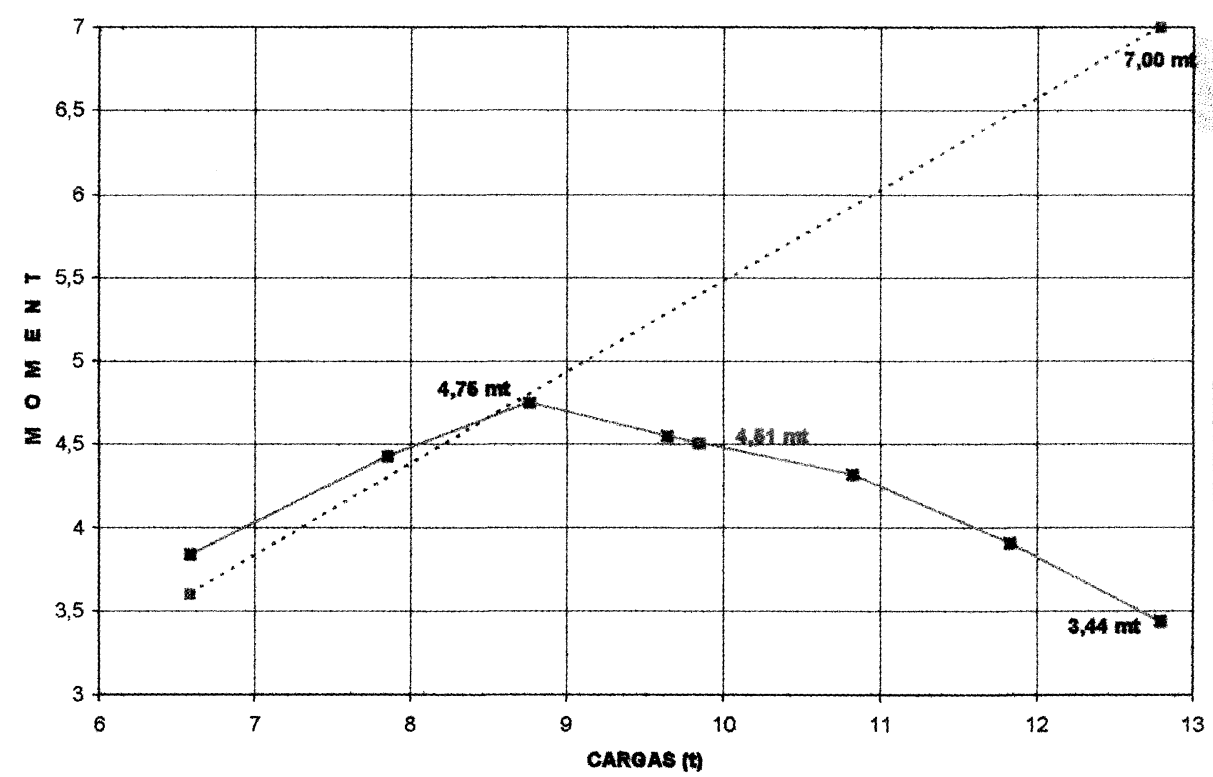

$81=0,68$

Figura 10.- Comparación redistribución obtenida en ensayo en losas de igual armado con aceros de distinta ductilidad. ARMADO $7 \phi 10 \mathrm{~mm}$. 

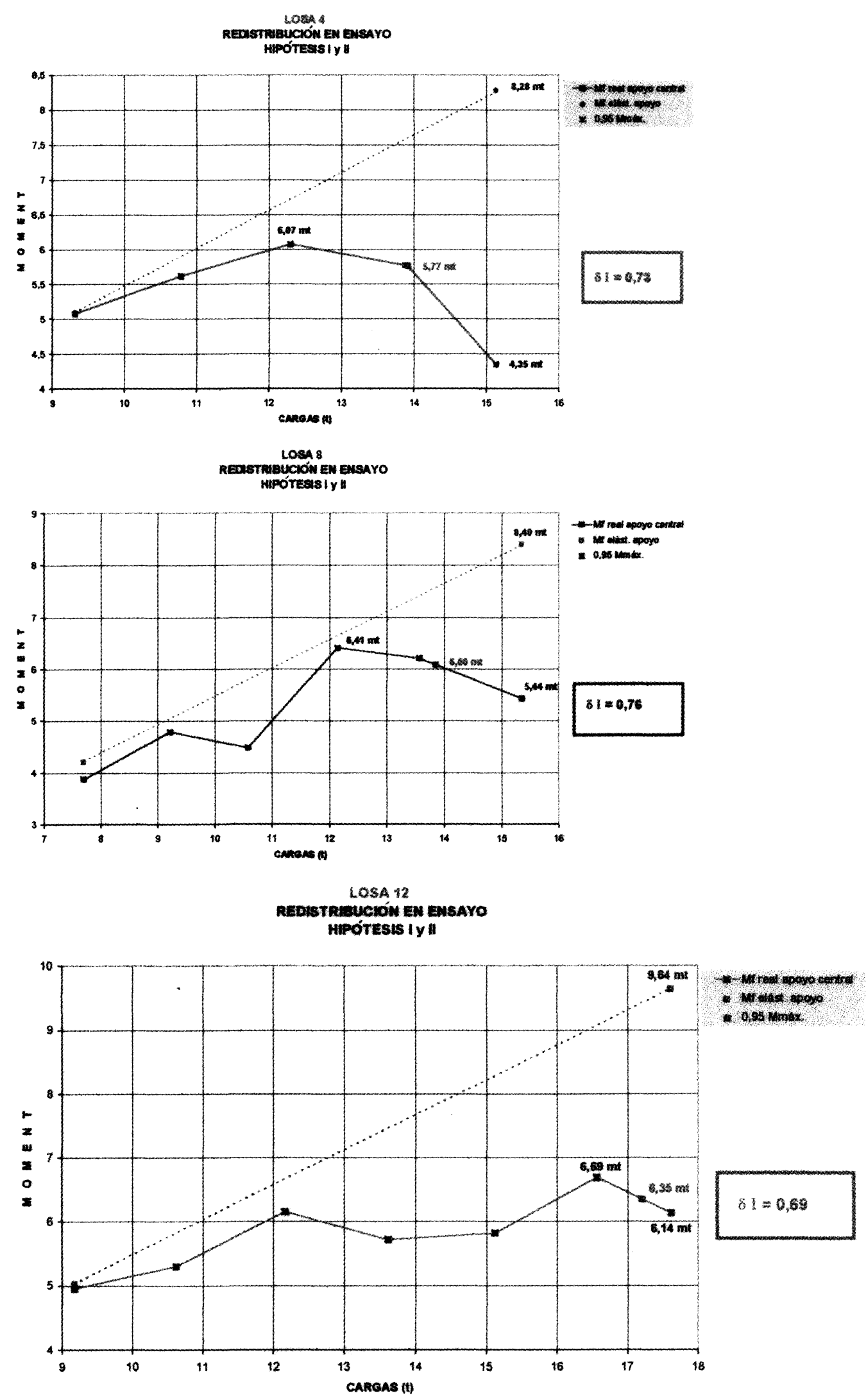

Figura 11.- Comparación redistribución obtenida en ensayo en losas de igual armado con aceros de distinta ductilidad. ARMADO $7 \phi 12 \mathrm{~mm}$. 

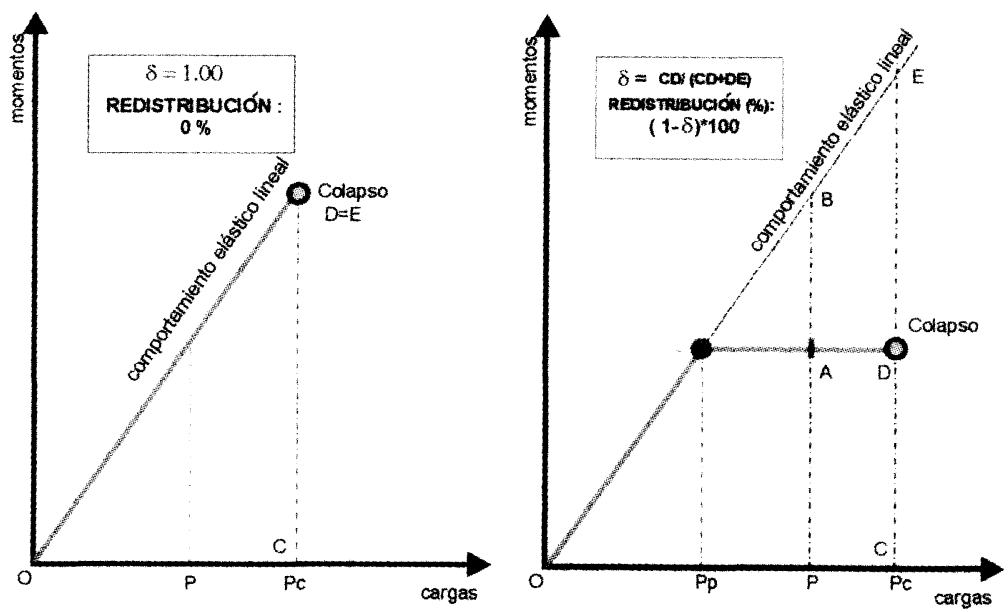

COMPORTAMIENTO ELASTO-PLÁSTICO PERFECTO

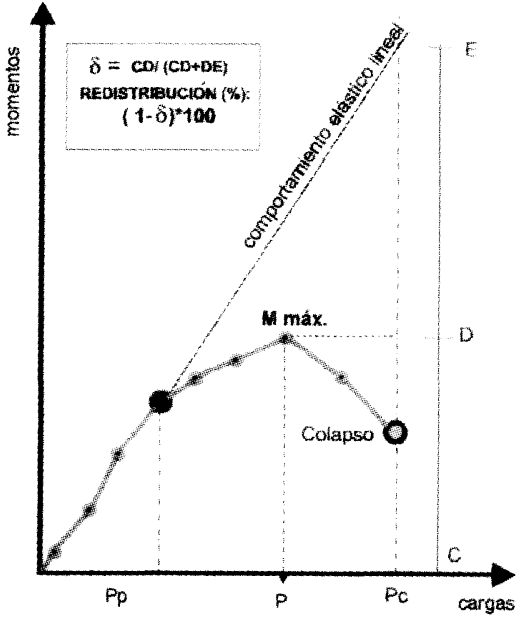

COMPORTAMIENTO REAL EN ENSAYO

Figura 12.- Concepto de redistribución real en ensayo.

das con las diferentes medidas de la ductilidad del acero empleado, se obtienen los gráficos de las figuras 13 y 14 . Se han considerado cuatro factores representativos del nivel de ductilidad de las armaduras: el Factor de Energía Plástica, el Factor de Ductilidad, el alargamiento bajo carga máxima $e_{\text {máx }}$ y la relación $\mathrm{f}_{\mathrm{s}} / \mathrm{f}_{\mathrm{y}}$. Todas, excepto la relación $f_{s} / f_{y}$, manifiestan el mismo tipo de tendencia en su relación con la redistribución alcanzada y que se refleja en las figuras citadas. La relación $\mathrm{f}_{\mathrm{s}} / \mathrm{f}_{\mathrm{y}}$, aunque manifiesta una cierta relación creciente con la redistribución, ésta no está bien definida, como puede verse en la figura 14 .

\section{Relación ductilidad vs seguridad frente al colapso}

Este es un aspecto de indudable interés como parte de este trabajo ya que se manifiesta una relación clara entre ductilidad y seguridad real frente al colapso.

En una primera aproximación al tema, se constata que, sin hacer otras consideraciónes, para cada trío de losas de la misma cuantía, las cargas reales más altas de colapso obtenidas en ensayo se corresponden con las armaduras de ductilidad más elevada, excepto en los casos de armado con la cuantía mínima $(0,15 \%)$ que tienen un comportamiento imprevisible y muy disperso (ver cuadro II).

Es evidente que esta comparación directa de las cargas reales de colapso, aunque proporciona una primera indicación, precisa un tratamiento más afinado para poder obtener conclusiones generales.

Una forma de tratar el tema es, como ya se ha apuntado, mediante la comparación de las cargas reales de colapso obtenidas experimentalmente con las cargas teóricas de cálculo obtenidas mediante las fórmulas de comproba- ción de secciones sometidas a flexión simple habitualmente empleadas, de acuerdo con lo ya expuesto en el apdo. 5. Con ello, se obtiene lo que podría llamarse coeficiente real de seguridad frente al colapso, ya que se conoce la magnitud de la relación entre la carga real que colapsa la estructura, frente a la nominal obtenida mediante el cálculo seccional en rotura empleado habitualmente en el proceso de dimensionamiento y armado de secciones. Para que la comparación sea adecuada, en las fórmulas de comprobación a flexión simple deberán introducirse los valores reales de las resistencias de los materiales obtenidos mediante los oportunos ensayos, tanto del hormigón mantenido en iguales condiciones que la estructura como, del acero introducido como armadura en la misma. En las fórmulas de comprobación, que más abajo se exponen, se introducen, por lo tanto, los valores reales del límite elástico del acero y de la resistencia a compresión del hormigón empleados en cada losa (ver cuadro I). De igual forma, estas resistencias de los materiales no se afectan de ningún coeficiente de minoración, es decir $\gamma_{\mathrm{s}}=0 \quad$ y $\gamma_{\mathrm{c}}=0$

Con estos valores, se calcula para cada losa el valor del momento que agota teóricamente la sección más solicitada (en este caso, la sección del apoyo central) y, con él, es inmediato el cálculo de la carga que le produce en el esquema estructural adoptado, bajo la hipótesis de comportamiento elástico lineal y posterior aplicación de la redistribución de momentos adoptada en este caso y que corresponde a igualar los momentos de vano y apoyo.

La comparación de los valores de las cargas de colapso realmente alcanzadas en ensayo con los valores de las cargas teóricas calculadas según lo expuesto, Preal/Pteor, proporciona un indicativo de la seguridad real de la estruc- 

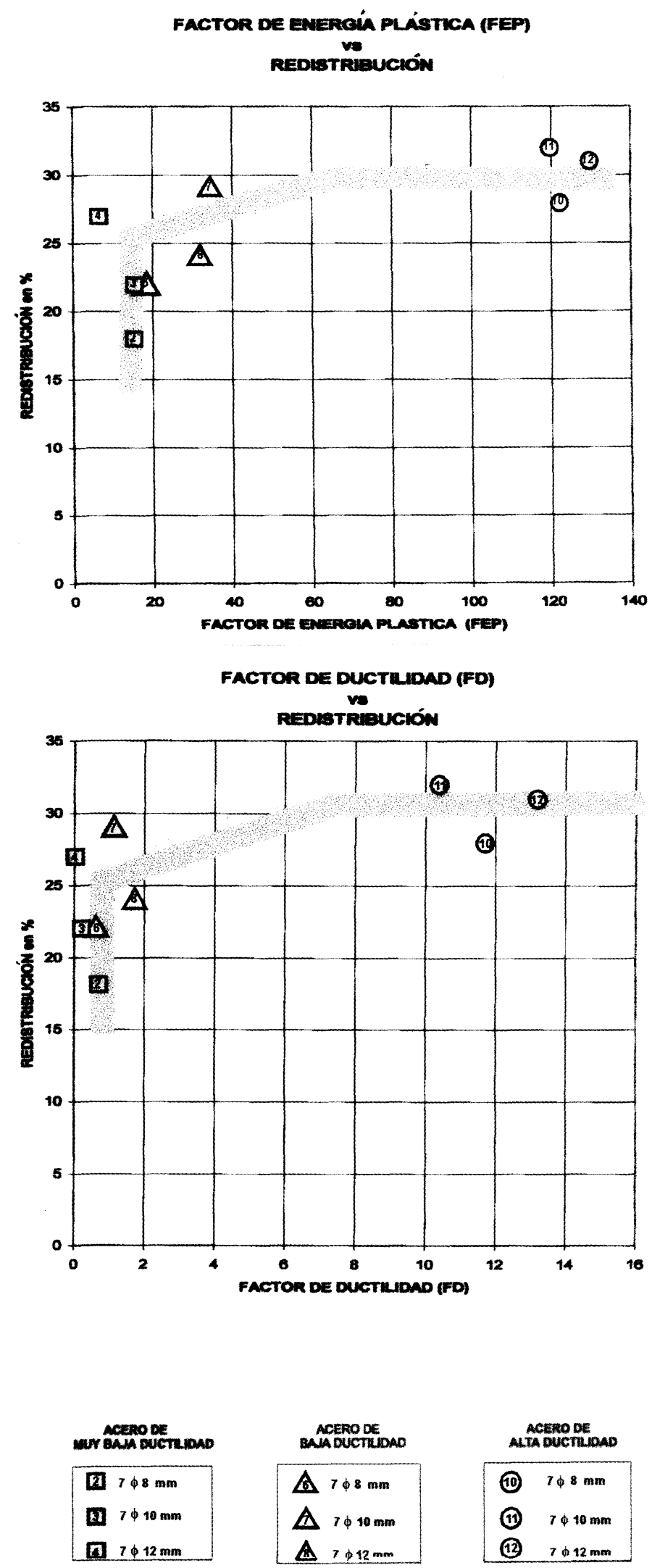

Figura 13.- Relación distribución vs. ductilidad (Factor de Energía Plástica "FEP" y Factor de Ductilidad "FD"). 

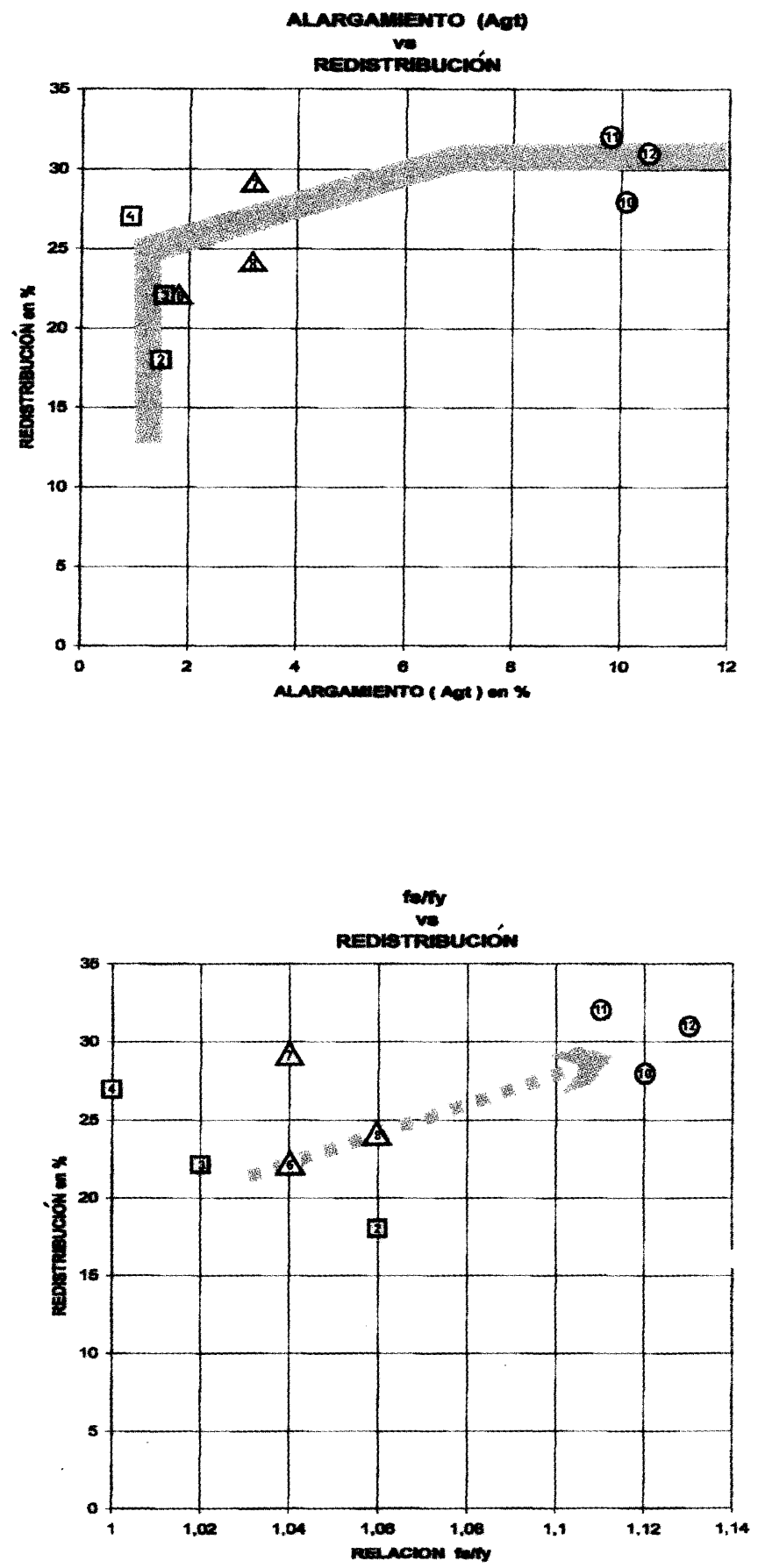

\begin{tabular}{|c|c|c|c|}
\hline wr MeEna be & $\begin{array}{l}\text { ACERTO DE } \\
\text { BANA DUCTIDAO }\end{array}$ & \multicolumn{2}{|c|}{ ACERTO OEE } \\
\hline [2] $7 \phi 8 \mathrm{~mm}$ & A $78 \mathrm{~mm}$ & (10) & $7 \phi \mathrm{mm}$ \\
\hline [] $7 \phi 10 \mathrm{~mm}$ & A. $70 \mathrm{~mm}$ & (11) & $7 \phi 10 \mathrm{~mm}$ \\
\hline [] $7612 \mathrm{~mm}$ & $\Delta 7.12 \mathrm{~mm}$ & (12) & $712 \mathrm{~mm}$ \\
\hline
\end{tabular}

Figura 14.- Relación distribución real vs.parámetros clásicos de ductilidad (alargamiento Agt y relación fs/fy). 
tura, independientemente de los coeficientes de seguridad que introduzcamos (minoración de las resistencias de los materiales y mayoración de las cargas).

Las fórmulas de comprobación empleadas para el cálculo en cada losa de los momentos últimos de las secciones más solicitadas son las siguientes:

INSTRUCCIÓN EH 91: Anejo 7 - Capítulo 3 - Apdo. 3.1 .2

$M_{u}=U_{s 1}\left(1-U_{s 1} /\left(2 U_{c}\right)\right) d$, siendo

$\mathrm{U}_{\mathrm{s} 1}=$ Capacidad mecánica de la armadura $=\mathrm{A}_{\mathrm{s}} \mathrm{f}_{\mathrm{y}}$

$\mathrm{U}_{\mathrm{c}}=$ Capacidad mecánica de la sección útil de hormigón $=\mathrm{f}_{\mathrm{c}} \mathrm{bd}$

$\mathrm{d}=$ Canto útil de la sección

INSTRUCCIÓN EHE : Anejo 8-Apdo. 3.2

$M_{u}=0,24 U_{v} d^{\prime}\left(U_{v}-U_{s}\right)\left(1,5 U_{s}\right) /\left(0,6 U_{v}\right)^{\wedge} 2$, siendo

$\mathrm{v}=2 \mathrm{U}_{0} \mathrm{~d}^{\prime} / \mathrm{d} ; \mathrm{U}_{0}=0,85 \mathrm{f}_{\mathrm{c}} \mathrm{bd} ; \mathrm{U}_{\mathrm{s}}=\mathrm{A}_{\mathrm{s}} \mathrm{f}_{\mathrm{y}}$

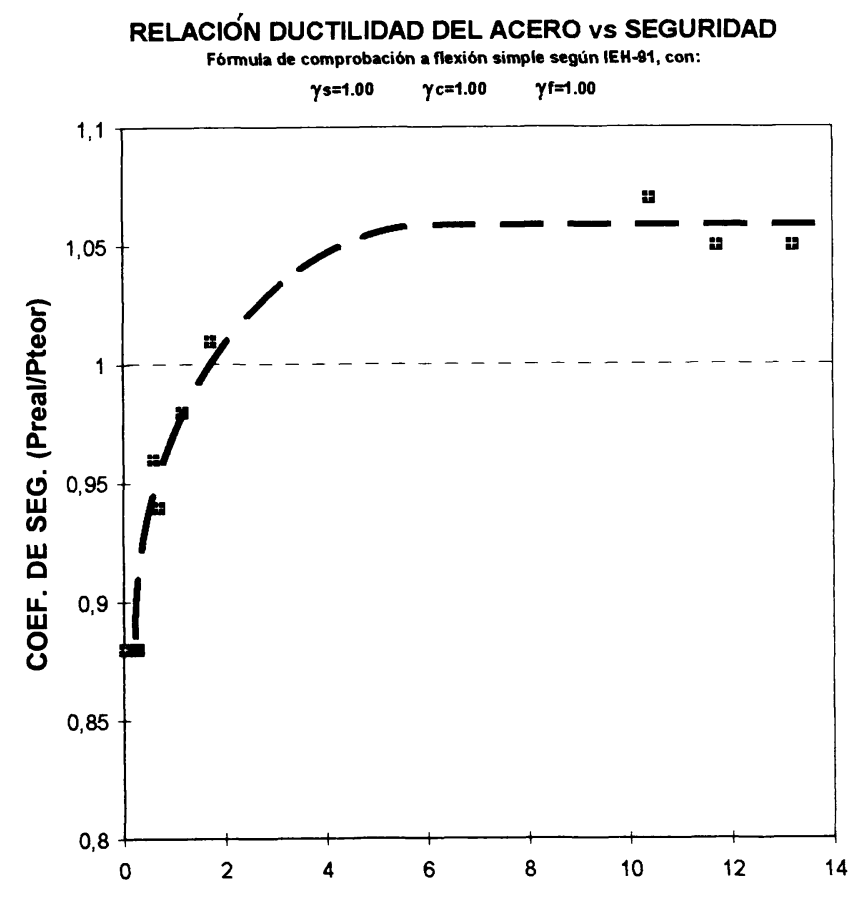

FACTOR DE DUCTILIDAD DEL ACERO F. D.)

Figura 15

(c) Consejo Superior de Investigaciones Científicas Licencia Creative Commons 3.0 España (by-nc)
En la figura 15 se expone la relación entre la ductilidad del acero y el cociente $\mathrm{P}_{\text {real }} / \mathrm{P}_{\text {teor }}$, siendo la $\mathrm{P}_{\text {teor }}$ obtenida a través de la fórmula de la $\mathrm{IEH}-91$, para las doce losas ensayadas con la tendencia que dicha relación presenta.

En la figura 16 se expone, igualmente, la misma relación, pero con la $\mathrm{P}_{\text {teor }}$ obtenida a través de la fórmula de la $\mathrm{EHE}$. En este caso, se observa un comportamiento más disperso que en el anterior, pero igualmente se manifiesta una tendencia creciente.

Las figuras 17 a 26 exponen la misma relación entre ductilidad de las armaduras y seguridad real, pero para cada trío de losas de igual cuantía, de forma que puedan compararse los casos en los que sólo ha cambiado la ductilidad de la armadura, manteniéndose las mismas cuantías y diámetros de las barras.

De esta forma se pueden conocer, por una lado, la forma general en la que se produce la relación ductilidad vs. seguridad (figuras 17 y 18) y, por otro, cuantificar los incrementos de seguridad para cada cuantía en función del aumento de la ductilidad. Ambos objetivos se resumen en el apdo. 8 siguiente.

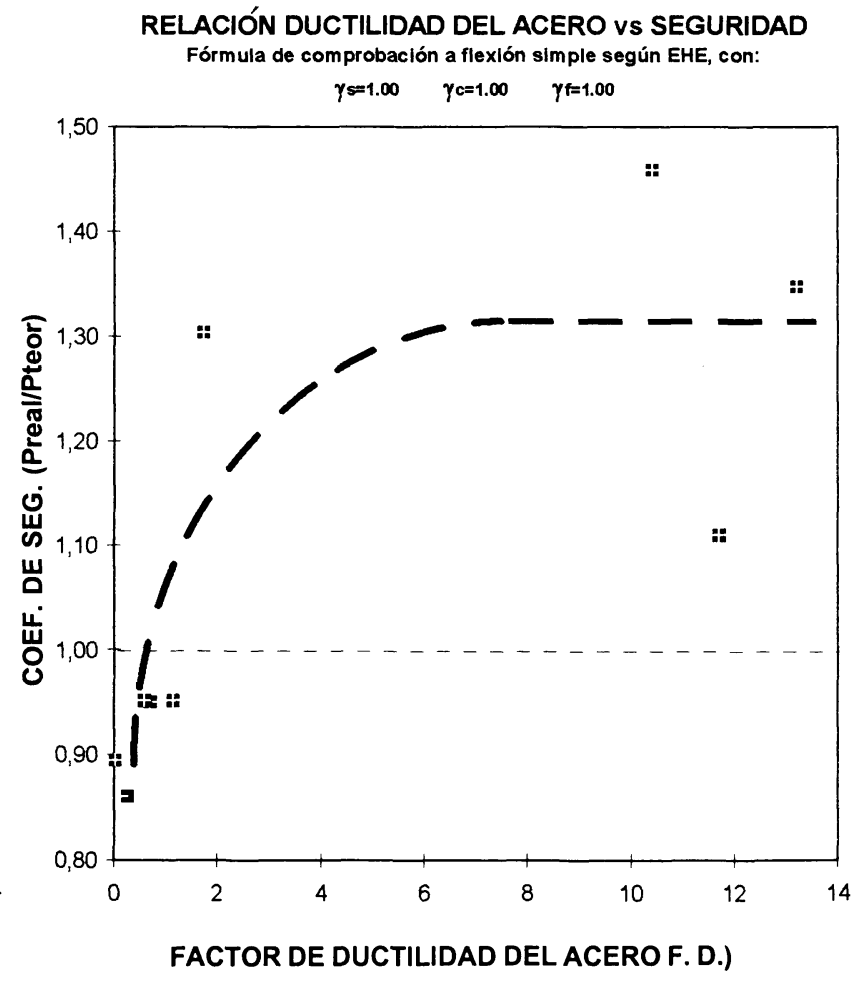

Figura 16 

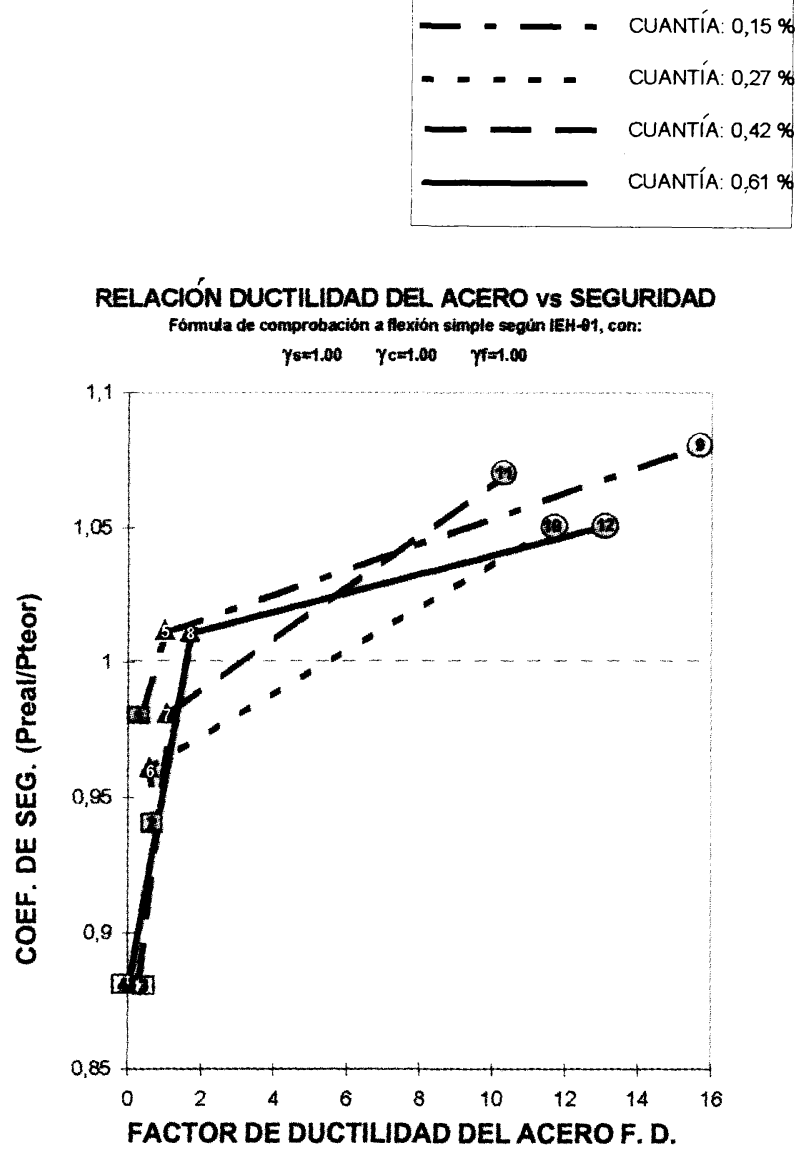

Figura 17

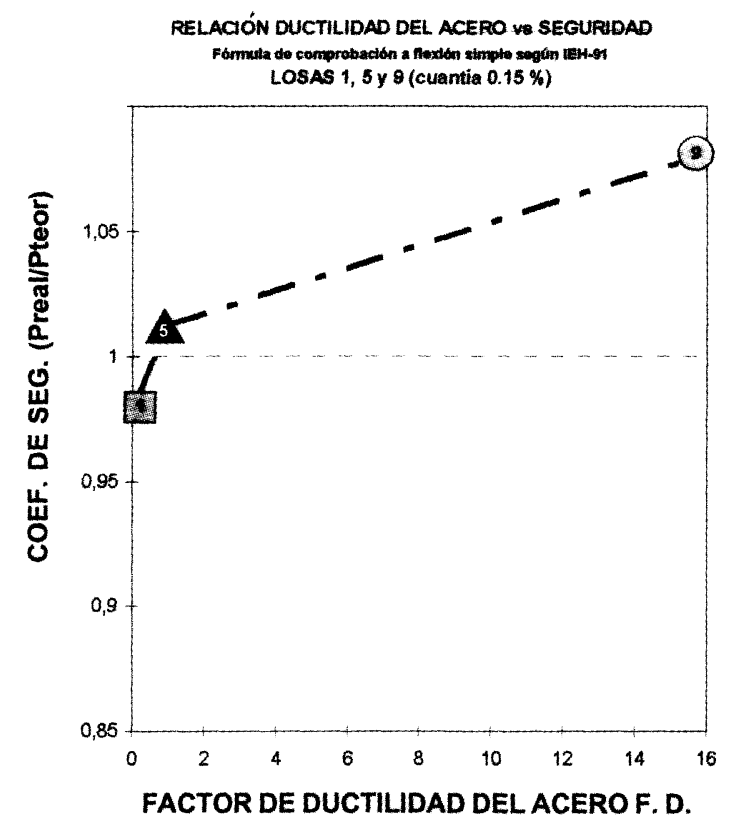

Figura 19

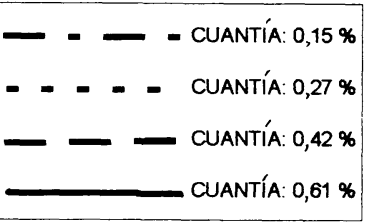

RELACIÓN DUCTILIDAD DEL ACERO vs SEGURIDAD Fómula de comprobación a flexion simple segün EHE, con: $\gamma_{s=1.00} \gamma_{c=1.00} \quad \gamma_{\text {rat }} .00$

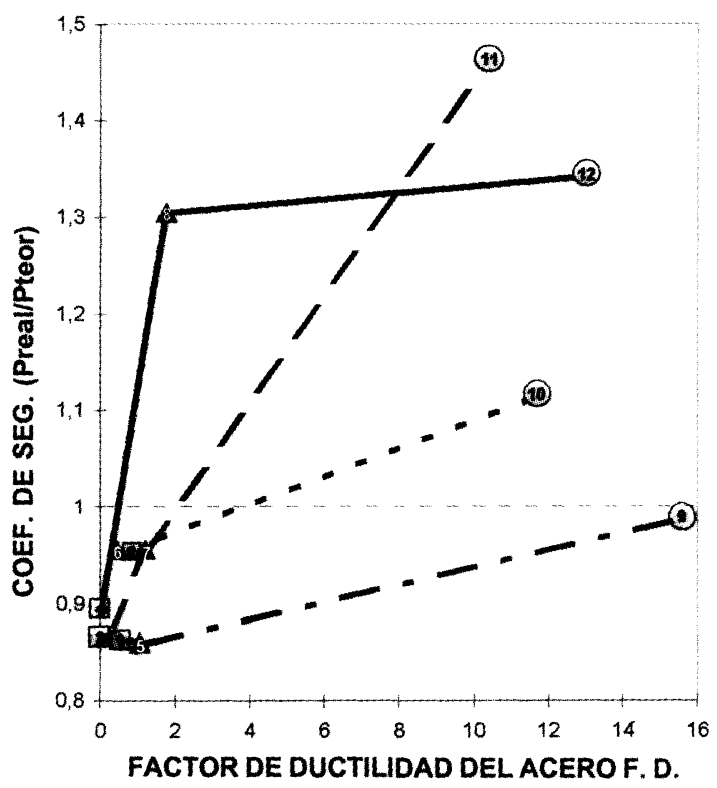

Figura 18

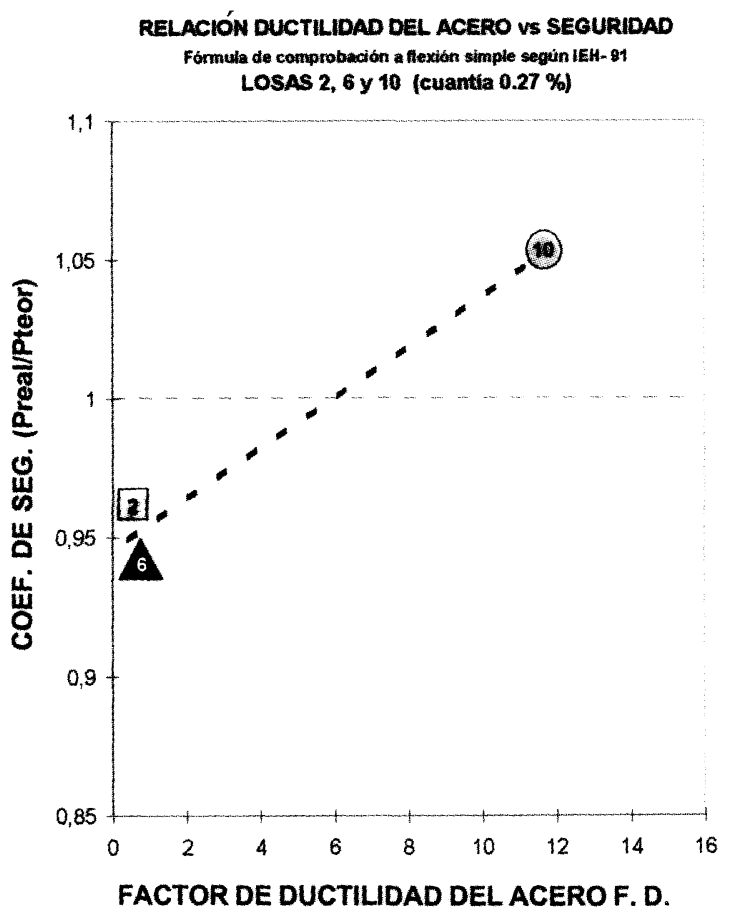

Figura 20 


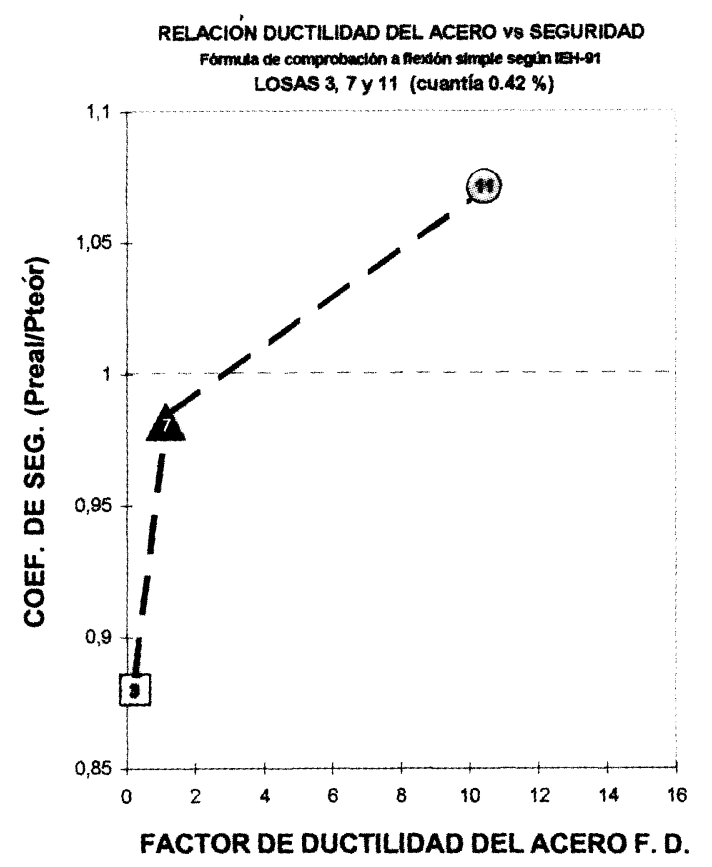

Figura 21

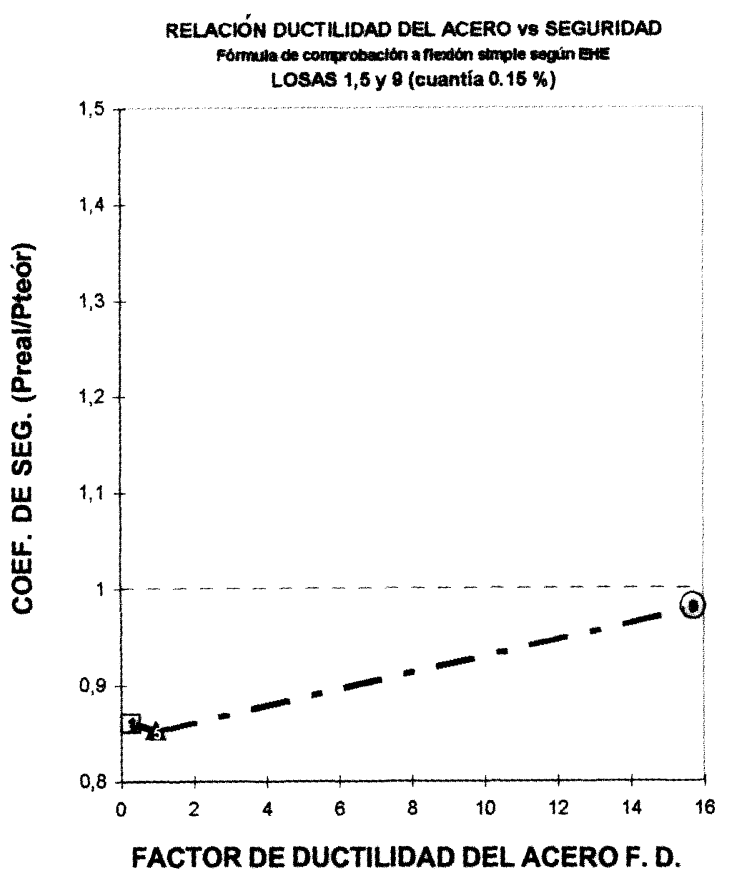

Figura 23

(c) Consejo Superior de Investigaciones Científicas Licencia Creative Commons 3.0 España (by-nc)

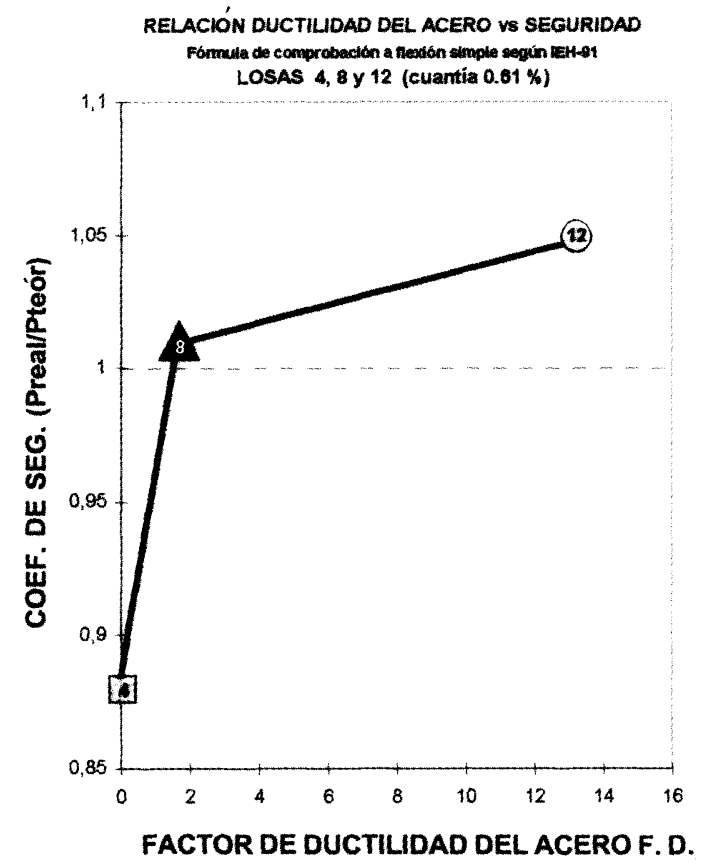

Figura 22

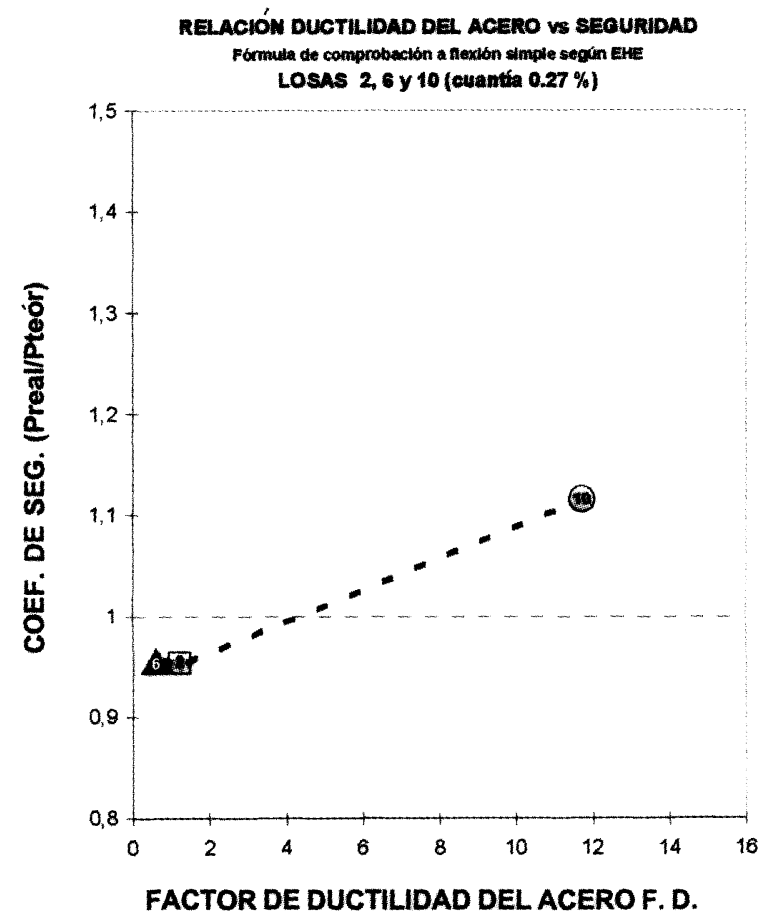

Figura 24 


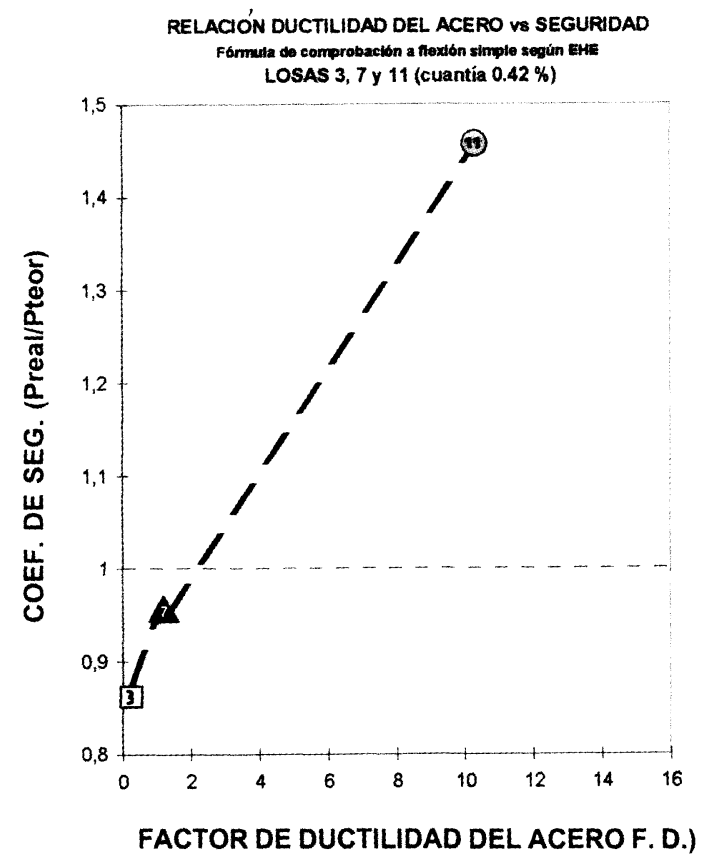

Figura 25

\section{Conclusiones}

A)Para cuantías muy bajas, como la correspondiente a las losas 1,5 y 9 (cuantía del 0,15\%) el comportamiento es impredecible con roturas frágiles y muy poco aviso previo. Aun así, con esta cuantía mínima, las losas armadas con acero de alta ductilidad proporcionan una seguridad superior a las armadas con aceros de baja ductilidad. Este aumento de la seguridad real se cifra entre un $10 \%$ y un $14 \%$.

B)La capacidad de redistribución aumenta con el incremento de la ductilidad de las armaduras dispuestas. Para valores del Factor de Ductilidad inferiores a 8, el descenso en la capacidad de redistribución es muy apreciable, de forma que con los aceros de muy baja y baja ductilidad no se alcanzan los niveles de redistribución previstos.

C)En términos generales, la ductilidad del acero de las armaduras influye de manera decisiva en el nivel de seguridad real frente al colapso, de forma que, a mayor ductilidad, mayor seguridad.

La tendencia general de comportamiento de esta relación es muy similar, como parece lógico, a la manifestada por

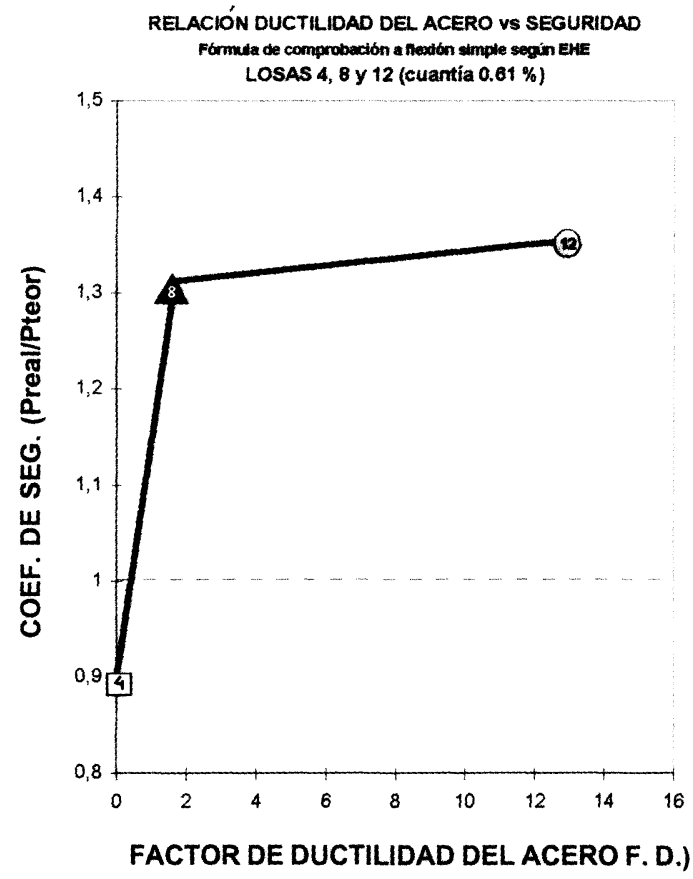

Figura 26

la relación ductilidad vs. redistribución, con un descenso de la seguridad real para valores del factor de ductilidad inferiores a 8.

Este nivel de ductilidad (FD alrededor de 8) corresponde, de acuerdo con la figura 4, a los aceros denominados de alta ductilidad, con unos parámetros mínimos $\varepsilon_{\text {máx }}>6 \%$ $y$ una relación $f_{s} / f_{y}>1,15$.

Los aceros con ductilidades inferiores, proporcionan unos niveles de seguridad claramente decrecientes.

A título de ejemplo, para las losas ensayadas con cuantías superiores a la mínima, el incremento de la seguridad real debida al empleo de acero de alta ductilidad puede llegar a alcanzar, si aplicamos la IEH 91, valores entre un $10 \%$ y $19 \%$, según la cuantía, en relación con los obtenidos con aceros de inferior ductilidad.

La aplicación de la formulación de la EHE, proporciona un incremento de seguridad real para estos aceros dúctiles en relación con los de baja ductilidad, situado entre un $17 \%$ y un $52 \%$. 